\author{
Economics Working Paper Series
}

2019/016

\title{
A Doubly Corrected Robust Variance Estimator for Linear GMM
}

Jungbin Hwang, Byunghoon Kang and Seojeong Lee

The Department of Economics

Lancaster University Management School

Lancaster LA1 4YX

UK provided that full acknowledgement is given. 


\title{
A Doubly Corrected Robust Variance Estimator for Linear GMM
}

\author{
Jungbin Hwang* $\quad$ Byunghoon Kang ${ }^{\dagger}$ \\ University of Connecticut Lancaster University \\ Seojeong Lee \\ University of New South Wales
}

August 12, 2019§

\begin{abstract}
We propose a new finite sample corrected variance estimator for the linear generalized method of moments (GMM) including the one-step, two-step, and iterated estimators. Our formula additionally corrects for the over-identification bias in variance estimation on top of the commonly used finite sample correction of Windmeijer (2005) which corrects for the bias from estimating the efficient weight matrix, so is doubly corrected. Formal stochastic expansions are derived to show the proposed double correction estimates the variance of some higher-order terms in the expansion. In addition, the proposed double correction provides robustness to misspecification of the moment condition. In contrast, the conventional variance estimator and the Windmeijer correction are inconsistent under misspecification. That is, the proposed double correction formula provides a convenient way to obtain improved inference under correct specification and robustness against misspecification at the same time.
\end{abstract}

\section{Introduction}

The generalized method of moments (GMM) estimators (Hansen, 1982) are widely used in economics. Among the class of GMM estimators, the efficient GMM has the smallest asymptotic variance which can be obtained via a two-step procedure. However, researchers have found that the standard error of the two-step efficient GMM is often severely downward biased. To solve this problem, Windmeijer (2005) proposed a finite sample bias corrected standard error formula for the

\footnotetext{
*Email: jungbin.hwang@uconn.edu

${ }^{\dagger}$ Kang acknowledges the research support from Lancaster University Management School (Research Pump-Prime Grants ECA6396), Email: b.kang1@lancaster.ac.uk

${ }^{\ddagger}$ Lee acknowledges that this research was supported under the Australian Research Council Discovery Early Career Reserach Award (DECRA) funding scheme (project number DE170100787), Email: jay.lee@unsw.edu.au

${ }^{\S}$ We thank Frank Windmeijer, Atsushi Inoue, Bruce Hansen, Frank Kleibergen, Whitney Newey, Richard Smith, and participants at ANZESG2019, ESAM2019, SETA2019, NASMES2019, and IPDC2019 for helpful comments and suggestions.
} 
two-step linear GMM. Specifically, his formula corrects for the bias arising from using the efficient weight matrix being evaluated at an estimate, rather than the true value. The correction formula (the Windmeijer correction, hereinafter) has been routinely used in practice. ${ }^{1}$

However, the Windmeijer correction does not take into account for the over-identification bias, which is another important source of bias in the GMM standard error. The over-identification bias arises from the fact that the over-identified sample moment condition is nonzero while it converges in probability to zero under correct specification.

We propose a new finite sample correction which takes into account for the over-identification bias for the variance of the linear one-step, two-step, and iterated GMM estimators. For one-step GMM such as the two-stage least squares (2SLS) estimators, the proposed finite sample correction is new as the Windmeijer correction does not cover the one-step GMM. For two-step and iterated GMM, the proposed correction improves upon the Windmeijer correction by additionally correcting for the over-identification bias. Thus, we doubly correct the finite sample bias of the linear GMM variance estimator.

We provide an additional formal justification of the proposed double correction by deriving the stochastic expansions of the GMM estimators under local misspecification where the moment condition is modeled as a local drifting sequence around zero. The result shows that the double correction estimates the (co)variance of some higher-order terms which increase with the overidentification bias. Rothenberg (1984) provides a general treatment of the stochastic expansion and Newey and Smith (2004) derive the stochastic expansion of GMM and the generalized empirical likelihood (GEL) estimators under correct specification.

The order of our double correction equals the order of the sample moment condition. Under correct specification or local misspecification, these terms are $O_{p}\left(n^{-1 / 2}\right)$ so that the double correction is a finite-sample correction for the variance. However, the double correction terms become $O_{p}(1)$ when the moment condition is (globally) misspecified because the sample moment condition is $O_{p}(1)$. The conventional variance estimator and the Windmeijer correction omit these $O_{p}(1)$ terms under misspecification. This implies that the conventional variance estimator and the Windmeijer correction are inconsistent, while our doubly corrected variance estimator is consistent regardless of whether the moment condition model is (locally or globally) misspecified or not.

Since the doubly corrected variance estimators are robust to misspecification, it is not surprising that the formulas coincides with the misspecification-robust variance estimator in Lee (2014) for the one-step and two-step GMM and B. Hansen and Lee (2019) for the iterated GMM. Indeed the simulation results reported in those papers show that the misspecification-robust variance estimator often performs better than the conventional variance estimator under correct specification. This paper provides an answer to this seemingly puzzling result by taking an alternative path to obtain the identical variance estimator formula.

From a practical point of view, the equivalence between the misspecification-robust formula and the doubly corrected formula implies that accurate inference under correct specification and

\footnotetext{
${ }^{1}$ More than 4,700 citations according to Google Scholar on Aug 5, 2019.
} 
robust inference under misspecification can be achieved simultaneously, without knowing whether the moment condition is correctly specified or not. Moreover, it can be easily implemented to obtain more accurate $t$ tests and confidence intervals (smaller errors in the size and the coverage) by bootstrapping the $t$ statistic studentized by the doubly corrected variance estimator. Lee (2014) shows that this bootstrap procedure is robust to misspecification and does not require an ad hoc correction in the bootstrap sample called recentering.

The finite sample correction of the proposed formula and the Windmeijer formula works for linear models. For nonlinear models, the order of the remainder term is the same as the correction terms, so that the corrections do not necessarily provide improvements under correct specification. Even for nonlinear models, however, the doubly corrected variance estimator is still consistent for the asymptotic variance of the GMM estimator under misspecification. So the robustness property holds for both linear and nonlinear models.

Robust inference with possibly misspecified moment condition models has gained considerable attention in the literature. Maasoumi and Phillips (1982) investigate the limiting distribution of inconsistent instrumental variable (IV) estimators. Guggenberger (2012) studies the behavior of the weak instrument robust tests under local misspecification. Lee (2018) shows that the moment condition is misspecified under treatment effect heterogeneity and proposes a robust variance estimator for 2SLS. Hall and Inoue (2003) derive the asymptotic distribution of GMM under misspecification. Schennach (2007) proposes an alternative GEL-type estimator robust to global misspecification. Otsu (2011) analyses moderate deviation behaviors of GMM. Kitamura, Otsu, and Evdokimov (2013) propose an estimator that achieves optimal minimax robust properties under local misspecification. Lee $(2014,2016)$ propose a robust nonparametric bootstrap procedure for GMM and GEL estimators. Hansen and Lee (2019) provide robust inference theory for the iterated GMM. Rotemberg (1983) and Andrews (2019) characterize the estimands of the linear GMM under misspecification. Andrews, Gentzkow, and Shapiro (2017) propose to measure the effect of model misspecification on the sensitivity of parameter estimates for the minimum distance estimators. Bonhomme and Weidner (2018) and Armstrong and Kolesár (2019) consider minimax and GMM inference under possible misspecification, respectively.

Finite sample properties of GMM estimators, including the iterated and the continuously updating (CU) GMM are investigated by Hansen, Heaton, and Yaron (1996). Bond and Windmeijer (2005) provide simulation evidence on the finite sample performance of the asymptotic and bootstrap tests based on GMM estimators. Hwang and Sun (2018) develop alternative asymptotics and compare the finite sample properties of the one-step and two-step GMM estimators for dependent observations.

Our doubly corrected robust variance estimators are generally different than the many instrument and many weak instrument robust variance estimators for IV, GMM, and GEL estimators proposed by Bekker (1994), Han and Phillips (2006), Newey and Windmeijer (2009), and Evdokimov and Kolesár (2018). Since our double correction formula does not use the many (weak) instruments asymptotics, it is not robust under such sequences. 
The remainder of the paper is organized as follows. Section 2 reviews the Windmeijer correction. Section 3 proposes the doubly corrected variance estimator. Section 4 derives the stochastic expansion of the one-step and two-step GMM estimators under local misspecification and shows that the double correction estimates the variance of some higher-order terms in the expansion. Section 5 shows that the doubly corrected variance estimator is misspecification-robust. Section 6 discusses the iterated GMM and the CU GMM. Section 7 derives the double correction formula for cross-sectional IV and the difference GMM. Finally Section 8 provides extensive simulation results comparing the double correction and other variance estimators. All the proofs are collected in the Appendix.

\section{Finite Sample Correction of Windmeijer (2005)}

Suppose that we observe a sequence of i.i.d. random vectors $X_{i} \in \mathbb{R}^{d_{x}}$ for $i=1, \ldots, n$. Let $g\left(X_{i}, \theta\right)$ be a $q \times 1$ moment function where $\theta$ is a $k \times 1$ parameter vector. We assume $q>k$ so that the model is over-identified and $g\left(X_{i}, \theta\right)$ is linear in parameter. When the model is just-identified $(q=k)$ the correction terms are zero and the analysis becomes trivial. The moment condition model is correctly specified if

$$
E\left[g\left(X_{i}, \theta_{0}\right)\right]=0
$$

for a unique $\theta_{0}$. Assume $E\left[\left|g\left(X_{i}, \theta_{0}\right)\right|^{2}\right]<\infty$. Then by the Lindeberg-Lévy central limit theorem

$$
\frac{1}{n} \sum_{i=1}^{n} g\left(X_{i}, \theta_{0}\right) \equiv g_{n}\left(\theta_{0}\right)=O_{p}\left(n^{-1 / 2}\right) .
$$

Thus, the sample moment condition converges in probability to zero at the rate of $n^{-1 / 2}$ under correct specification (1). This will be used in determining the order of higher-order terms in Sections 2 and 3. We also assume standard regularity conditions for consistency and asymptotic normality of the GMM estimators, e.g., the conditions of Theorems 2.6 and 3.4 of Newey and McFadden (1994).

The one-step GMM estimator is defined as

$$
\hat{\theta}_{1}=\underset{\theta \in \Theta}{\arg \min } g_{n}(\theta)^{\prime} W_{n}^{-1} g_{n}(\theta)
$$

where $W_{n}$ is a $q \times q$ positive definite weight matrix which takes the form of $n^{-1} \sum_{i=1}^{n} W\left(X_{i}\right)$ and $W\left(X_{i}\right)$ does not depend on any unknown parameter. Common choices of $W\left(X_{i}\right)$ are the identity matrix and $Z_{i} Z_{i}^{\prime}$ where $Z_{i}$ is the instrument vector in IV regressions. Let $W=E W_{n}$, a positive definite matrix of constants.

Taking $\hat{\theta}_{1}$ as a preliminary (initial) estimator, the two-step efficient GMM estimator is obtained by minimizing

$$
\hat{\theta}_{2}=\arg \min _{\theta \in \Theta} g_{n}(\theta)^{\prime}\left[\Omega_{n}\left(\hat{\theta}_{1}\right)\right]^{-1} g_{n}(\theta),
$$


where

$$
\Omega_{n}(\theta)=\frac{1}{n} \sum_{i=1}^{n} g\left(X_{i}, \theta\right) g\left(X_{i}, \theta\right)^{\prime} .
$$

Define $\Omega=\Omega\left(\theta_{0}\right)$ where $\Omega(\theta)=E \Omega_{n}(\theta)$. Since $\Omega_{n}\left(\hat{\theta}_{1}\right)$ is consistent for the asymptotic variance of the moment function the two-step GMM is efficient.

We also define an infeasible two-step GMM estimator $\tilde{\theta}_{2}$ using $\left[\Omega_{n}\left(\theta_{0}\right)\right]^{-1}$ as the weight matrix:

$$
\tilde{\theta}_{2}=\arg \min _{\theta \in \Theta} g_{n}(\theta)^{\prime}\left[\Omega_{n}\left(\theta_{0}\right)\right]^{-1} g_{n}(\theta)
$$

Investigating the limiting behavior of $\sqrt{n}\left(\tilde{\theta}_{2}-\theta_{0}\right)$ will help us understand the higher-order behavior of the feasible two-step estimator $\sqrt{n}\left(\hat{\theta}_{2}-\theta_{0}\right)$.

Let $G\left(X_{i}\right)=\partial g\left(X_{i}, \theta\right) / \partial \theta^{\prime}$. Note that it does not depend on $\theta$ due to linearity. Define $G_{n}=$ $n^{-1} \sum_{i=1}^{n} G\left(X_{i}\right)$ and $G=E G_{n}$. By the first-order Taylor expansion, the first-order condition (FOC) of the (feasible) two-step GMM can be written as

$$
\begin{aligned}
0 & =G_{n}^{\prime}\left[\Omega_{n}\left(\hat{\theta}_{1}\right)\right]^{-1} g_{n}\left(\hat{\theta}_{2}\right) \\
& =G_{n}^{\prime}\left[\Omega_{n}\left(\hat{\theta}_{1}\right)\right]^{-1}\left[g_{n}\left(\theta_{0}\right)+G_{n}\left(\hat{\theta}_{2}-\theta_{0}\right)\right],
\end{aligned}
$$

and so we have

$$
\sqrt{n}\left(\hat{\theta}_{2}-\theta_{0}\right)=-\left\{G_{n}^{\prime}\left[\Omega_{n}\left(\hat{\theta}_{1}\right)\right]^{-1} G_{n}\right\}^{-1} G_{n}^{\prime}\left[\Omega_{n}\left(\hat{\theta}_{1}\right)\right]^{-1} \sqrt{n} g_{n}\left(\theta_{0}\right)
$$

Using a similar expansion, we can get

$$
\sqrt{n}\left(\tilde{\theta}_{2}-\theta_{0}\right)=-\left\{G_{n}^{\prime}\left[\Omega_{n}\left(\theta_{0}\right)\right]^{-1} G_{n}\right\}^{-1} G_{n}^{\prime}\left[\Omega_{n}\left(\theta_{0}\right)\right]^{-1} \sqrt{n} g_{n}\left(\theta_{0}\right)
$$

for the infeasible two-step GMM and

$$
\sqrt{n}\left(\hat{\theta}_{1}-\theta_{0}\right)=-\left(G_{n}^{\prime} W_{n}^{-1} G_{n}\right)^{-1} G_{n}^{\prime} W_{n}^{-1} \sqrt{n} g_{n}\left(\theta_{0}\right)
$$

for the one-step GMM.

Asymptotically (6) and (7) have the same limiting distribution so that using $\Omega_{n}\left(\hat{\theta}_{1}\right)$ instead of $\Omega_{n}\left(\theta_{0}\right)$ does not affect the first-order asymptotic analysis. However, by expanding $\Omega_{n}\left(\hat{\theta}_{1}\right)$ around $\theta_{0}$ and using (8), Windmeijer (2005) shows that the extra finite sample variations caused by higherorder terms can be estimated and the accuracy of the variance estimate can be improved for linear moment condition models.

To see this, we use the first-order Taylor expansion of $\Omega_{n}\left(\hat{\theta}_{1}\right)$ in the right-hand side (RHS) of 
(6) around $\theta_{0}$ :

$$
\begin{aligned}
\sqrt{n}\left(\hat{\theta}_{2}-\theta_{0}\right) & =-\left\{G_{n}^{\prime}\left[\Omega_{n}\left(\theta_{0}\right)\right]^{-1} G_{n}\right\}^{-1} G_{n}^{\prime}\left[\Omega_{n}\left(\theta_{0}\right)\right]^{-1} \sqrt{n} g_{n}\left(\theta_{0}\right)+D_{n} \sqrt{n}\left(\hat{\theta}_{1}-\theta_{0}\right)+O_{p}\left(n^{-1}\right) \\
& =\sqrt{n}\left(\tilde{\theta}_{2}-\theta_{0}\right)+\underbrace{D_{n} \sqrt{n}\left(\hat{\theta}_{1}-\theta_{0}\right)}_{=O_{p}\left(n^{-1 / 2}\right)}+O_{p}\left(n^{-1}\right)
\end{aligned}
$$

where

$$
\begin{aligned}
& D_{n}=F_{1 n}+F_{2 n}, \\
& F_{1 n}=-\left.\frac{\partial\left\{G_{n}^{\prime}\left[\Omega_{n}(\theta)\right]^{-1} G_{n}\right\}^{-1}}{\partial \theta^{\prime}}\right|_{\theta=\theta_{0}} G_{n}^{\prime}\left[\Omega_{n}\left(\theta_{0}\right)\right]^{-1} g_{n}\left(\theta_{0}\right), \\
& F_{2 n}=-\left.\left\{G_{n}^{\prime}\left[\Omega_{n}\left(\theta_{0}\right)\right]^{-1} G_{n}\right\}^{-1} \frac{\partial G_{n}^{\prime}\left[\Omega_{n}(\theta)\right]^{-1} g_{n}\left(\theta_{0}\right)}{\partial \theta^{\prime}}\right|_{\theta=\theta_{0}}
\end{aligned}
$$

are $k \times k$ matrices. Since $g_{n}\left(\theta_{0}\right)=O_{p}\left(n^{-1 / 2}\right)$ both $F_{1 n}$ and $F_{2 n}$ are $O_{p}\left(n^{-1 / 2}\right)$. Combining these with the assumption that $\sqrt{n}\left(\hat{\theta}_{1}-\theta_{0}\right)=O_{p}(1)$, the second term in the above expansion (9) has the order of $O_{p}\left(n^{-1 / 2}\right)$, which is of a lower order than the last term. Thus, by taking into account for the variation caused by the $O_{p}\left(n^{-1 / 2}\right)$ term, the finite sample variance of $\sqrt{n}\left(\hat{\theta}_{2}-\theta_{0}\right)$ can be more accurately approximated. Note that the expansion (9) only holds for linear moment condition models.

The Windmeijer correction of the variance of $\sqrt{n}\left(\hat{\theta}_{2}-\theta_{0}\right)$ is obtained by

$$
\widehat{V}_{w}\left(\hat{\theta}_{2}\right)=\widetilde{V}\left(\hat{\theta}_{2}\right)+\widehat{D}_{n} \widetilde{V}\left(\hat{\theta}_{2}\right)+\widetilde{V}\left(\hat{\theta}_{2}\right) \widehat{D}_{n}^{\prime}+\widehat{D}_{n} \widetilde{V}\left(\hat{\theta}_{1}\right) \widehat{D}_{n}^{\prime}
$$

where $D[., j]$ denotes the $j$ th column of $D, \theta_{[j]}$ denotes the $j$ th element of $\theta$, and

$$
\begin{aligned}
\widetilde{V}\left(\hat{\theta}_{1}\right) & =\left(G_{n}^{\prime} W_{n}^{-1} G_{n}\right)^{-1}\left(G_{n}^{\prime} W_{n}^{-1} \Omega_{n}\left(\hat{\theta}_{1}\right) W_{n}^{-1} G_{n}\right)\left(G_{n}^{\prime} W_{n}^{-1} G_{n}\right)^{-1}, \\
\widetilde{V}\left(\hat{\theta}_{2}\right) & =\left\{G_{n}^{\prime}\left[\Omega_{n}\left(\hat{\theta}_{1}\right)\right]^{-1} G_{n}\right\}^{-1}, \\
\widehat{D}_{n}[., j] & =\left\{G_{n}^{\prime}\left[\Omega_{n}\left(\hat{\theta}_{1}\right)\right]^{-1} G_{n}\right\}^{-1} G_{n}^{\prime}\left\{\left.\left[\Omega_{n}\left(\hat{\theta}_{1}\right)\right]^{-1} \frac{\partial \Omega_{n}(\theta)}{\partial \theta_{[j]}}\right|_{\theta=\hat{\theta}_{1}}\left[\Omega_{n}\left(\hat{\theta}_{1}\right)\right]^{-1}\right\} g_{n}\left(\hat{\theta}_{2}\right), \\
\frac{\partial \Omega_{n}(\theta)}{\partial \theta_{[j]}} & =\Upsilon_{j}(\theta)+\Upsilon_{j}^{\prime}(\theta), \\
\Upsilon_{j}(\theta) & =\frac{1}{n} \sum_{i=1}^{n} g_{i}\left(X_{i}, \theta\right) \frac{\partial g_{i}\left(X_{i}, \theta\right)^{\prime}}{\partial \theta_{[j]}} .
\end{aligned}
$$

Since the estimate of $F_{1 n}$ equals to zero because of $0=G_{n}^{\prime}\left[\Omega_{n}\left(\hat{\theta}_{1}\right)\right]^{-1} g_{n}\left(\hat{\theta}_{2}\right)$ by the FOC, it does not appear in the variance estimator formula. The standard error is obtained by taking the diagonal elements of $\sqrt{\widehat{V}_{w}\left(\hat{\theta}_{2}\right) / n}$. 


\section{Double Correction}

The Windmeijer correction takes into account for the extra variability due to using an estimated parameter in the weight matrix. This correction is effective because $\widehat{D}_{n} \neq 0$, which is due to $g_{n}\left(\hat{\theta}_{2}\right) \neq 0$ in finite sample. In fact, $g_{n}(\theta) \neq 0$ for all $\theta$, which (trivially) implies $g_{n}\left(\theta_{0}\right) \neq 0$. We call this the over-identification bias, which is non-zero for any $n$ in general for over-identified models.

We show that the over-identification bias causes additional finite sample variability in (9). These additional terms are not considered in the Windmeijer correction (10). We propose alternative variance estimators that fully incorporate the additional variations induced by the over-identification bias. These variance estimators will replace $\widetilde{V}\left(\hat{\theta}_{2}\right)$ and $\widetilde{V}\left(\hat{\theta}_{1}\right)$ in (10) without affecting the order of finite sample corrections, leading to our doubly corrected variance estimator.

Assume that

$$
\begin{aligned}
G_{n}-G & =O_{p}\left(n^{-1 / 2}\right), \\
\operatorname{vec}\left(W_{n}-W\right) & =O_{p}\left(n^{-1 / 2}\right), \\
\operatorname{vec}\left(\Omega_{n}\left(\theta_{0}\right)-\Omega\right) & =O_{p}\left(n^{-1 / 2}\right),
\end{aligned}
$$

which hold under appropriate regularity conditions. Since $G^{\prime} \Omega^{-1} g=0$ by the population FOC and

$$
\left[\Omega_{n}\left(\theta_{0}\right)\right]^{-1}-\Omega^{-1}=-\Omega^{-1}\left(\Omega_{n}\left(\theta_{0}\right)-\Omega\right)\left[\Omega_{n}\left(\theta_{0}\right)\right]^{-1},
$$

we can write

$$
\begin{aligned}
& G_{n}^{\prime}\left[\Omega_{n}\left(\theta_{0}\right)\right]^{-1} g_{n}\left(\theta_{0}\right) \\
& =\underbrace{G^{\prime} \Omega^{-1} g_{n}\left(\theta_{0}\right)}_{=O_{p}\left(n^{-1 / 2}\right)}+\underbrace{\left(G_{n}-G\right)^{\prime} \Omega^{-1} g_{n}\left(\theta_{0}\right)-G^{\prime} \Omega^{-1}\left(\Omega_{n}\left(\theta_{0}\right)-\Omega\right) \Omega^{-1} g_{n}\left(\theta_{0}\right)}_{=O_{p}\left(n^{-1}\right)}+O_{p}\left(n^{-3 / 2}\right) .
\end{aligned}
$$

Using (15), the expansion of the infeasible two-step GMM (7) can be written as

$$
\begin{aligned}
\sqrt{n}\left(\tilde{\theta}_{2}-\theta_{0}\right)= & -\left\{G_{n}^{\prime}\left[\Omega_{n}\left(\theta_{0}\right)\right]^{-1} G_{n}\right\}^{-1}\left[G^{\prime} \Omega^{-1} \sqrt{n} g_{n}\left(\theta_{0}\right)\right. \\
& \left.+\sqrt{n}\left(G_{n}-G\right)^{\prime} \Omega^{-1} g_{n}\left(\theta_{0}\right)-G^{\prime} \Omega^{-1} \sqrt{n}\left(\Omega_{n}\left(\theta_{0}\right)-\Omega\right) \Omega^{-1} g_{n}\left(\theta_{0}\right)\right] \\
& +O_{p}\left(n^{-1}\right) .
\end{aligned}
$$

Similarly,

$$
\begin{aligned}
\sqrt{n}\left(\hat{\theta}_{1}-\theta_{0}\right)= & -\left\{G_{n}^{\prime} W_{n}^{-1} G_{n}\right\}^{-1}\left[G^{\prime} W^{-1} \sqrt{n} g_{n}\left(\theta_{0}\right)\right. \\
& \left.+\sqrt{n}\left(G_{n}-G\right)^{\prime} W^{-1} g_{n}\left(\theta_{0}\right)-G^{\prime} W^{-1} \sqrt{n}\left(W_{n}-W\right) W^{-1} g_{n}\left(\theta_{0}\right)\right] \\
& +O_{p}\left(n^{-1}\right),
\end{aligned}
$$


which simplifies to

$$
\sqrt{n}\left(\hat{\theta}_{1}-\theta_{0}\right)=-\left\{G_{n}^{\prime} G_{n}\right\}^{-1}\left[G^{\prime} \sqrt{n} g_{n}\left(\theta_{0}\right)+\sqrt{n}\left(G_{n}-G\right)^{\prime} g_{n}\left(\theta_{0}\right)\right]
$$

when $W_{n}=I$. From the above expansions it is clear that (i) to take into account for the overidentification bias we need to consider the extra variations from $\sqrt{n}\left(G_{n}-G\right)$ and $\sqrt{n}\left(\Omega_{n}(\theta)-\Omega\right)$ (or $\sqrt{n}\left(W_{n}-W\right)$ for the one-step GMM), and (ii) the order of the remainder term of the original expansion (9) is not changed.

Using the expansions (16)-(18) an (19)-(21), the expansion of the two-step GMM can be written as

$$
\begin{aligned}
\sqrt{n}\left(\hat{\theta}_{2}-\theta_{0}\right)= & -\left\{G_{n}^{\prime}\left[\Omega_{n}\left(\theta_{0}\right)\right]^{-1} G_{n}\right\}^{-1}\left[G^{\prime} \Omega^{-1} \sqrt{n} g_{n}\left(\theta_{0}\right)\right. \\
& \left.+\sqrt{n}\left(G_{n}-G\right)^{\prime} \Omega^{-1} g_{n}\left(\theta_{0}\right)-G^{\prime} \Omega^{-1} \sqrt{n}\left(\Omega_{n}\left(\theta_{0}\right)-\Omega\right) \Omega^{-1} g_{n}\left(\theta_{0}\right)\right] \\
& -D_{n}\left\{G_{n}^{\prime} W_{n}^{-1} G_{n}\right\}^{-1}\left[G^{\prime} W^{-1} \sqrt{n} g_{n}\left(\theta_{0}\right)\right. \\
& \left.+\sqrt{n}\left(G_{n}-G\right)^{\prime} W^{-1} g_{n}\left(\theta_{0}\right)-G^{\prime} W^{-1} \sqrt{n}\left(W_{n}-W\right) W^{-1} g_{n}\left(\theta_{0}\right)\right] \\
& +O_{p}\left(n^{-1}\right)
\end{aligned}
$$

In finite sample, $g_{n}\left(\theta_{0}\right) \neq 0$ because $g_{n}(\theta) \neq 0$ for all $\theta$, and this causes extra variations through the terms in (24) and (26). Similar to the Windmeijer correction, by taking into account for these (asymptotically negligible) terms in estimating the variance we can make more accurate inference.

Since $D_{n}=O_{p}\left(n^{-1 / 2}\right)$, the terms in (26) are $O_{p}\left(n^{-1}\right)$ multiplied by $D_{n}$, which is the same order with the remainder term. Thus, considering those terms in (26) does not necessarily provide finite sample corrections. However, including these terms are critical to get robustness to misspecification, which is shown in Section 5.

The expansion for the one-step GMM is (19)-(21) and those terms in (20) are $O_{p}\left(n^{-1 / 2}\right)$. Thus, by considering the finite sample variation caused by these terms provide more accurate inference. This correction for the one-step GMM is not considered in Windmeijer (2005) and is new.

The relationship between the expansions in (19)-(21) and (23)-(27), and their respective higherorder stochastic expansions will be explained in the next Section.

The doubly corrected variance estimator of $\sqrt{n}\left(\hat{\theta}_{2}-\theta_{0}\right)$ is

$$
\widehat{V}_{d c}\left(\hat{\theta}_{2}\right)=\widehat{V}\left(\hat{\theta}_{2}\right)+\widehat{D}_{n} \widehat{C}\left(\hat{\theta}_{1}, \hat{\theta}_{2}\right)+\widehat{C}\left(\hat{\theta}_{1}, \hat{\theta}_{2}\right)^{\prime} \widehat{D}_{n}^{\prime}+\widehat{D}_{n} \widehat{V}_{d c}\left(\hat{\theta}_{1}\right) \widehat{D}_{n}^{\prime}
$$

where

$$
\begin{aligned}
\widehat{V}\left(\hat{\theta}_{2}\right) & =\left(G_{n}^{\prime}\left[\Omega_{n}\left(\hat{\theta}_{1}\right)\right]^{-1} G_{n}\right)^{-1} \Sigma_{n}\left(\hat{\theta}_{2}, \Omega_{n}\left(\hat{\theta}_{1}\right)\right)\left(G_{n}^{\prime}\left[\Omega_{n}\left(\hat{\theta}_{1}\right)\right]^{-1} G_{n}\right)^{-1}, \\
\widehat{V}_{d c}\left(\hat{\theta}_{1}\right) & =\left(G_{n}^{\prime} W_{n}^{-1} G_{n}\right)^{-1} \Sigma_{n}\left(\hat{\theta}_{1}, W_{n}\right)\left(G_{n}^{\prime} W_{n}^{-1} G_{n}\right)^{-1}, \\
\widehat{C}\left(\hat{\theta}_{1}, \hat{\theta}_{2}\right) & =\left(G_{n}^{\prime} W_{n}^{-1} G_{n}\right)^{-1} \frac{1}{n} \sum_{i=1}^{n} m_{i}\left(\hat{\theta}_{1}, W_{n}\right) m_{i}\left(\hat{\theta}_{2}, \Omega_{n}\left(\hat{\theta}_{1}\right)\right)^{\prime}\left(G_{n}^{\prime}\left[\Omega_{n}\left(\hat{\theta}_{1}\right)\right]^{-1} G_{n}\right)^{-1},
\end{aligned}
$$


and

$$
\begin{aligned}
\Sigma_{n}\left(\theta, \Xi_{n}(\phi)\right)= & \frac{1}{n} \sum_{i=1}^{n} m_{i}\left(\theta, \Xi_{n}(\phi)\right) m_{i}\left(\theta, \Xi_{n}(\phi)\right)^{\prime}, \\
m_{i}\left(\theta, \Xi_{n}(\phi)\right)= & G_{n}^{\prime}\left[\Xi_{n}(\phi)\right]^{-1} g\left(X_{i}, \theta\right)+G\left(X_{i}\right)^{\prime}\left[\Xi_{n}(\phi)\right]^{-1} g_{n}(\theta) \\
& -G_{n}^{\prime}\left[\Xi_{n}(\phi)\right]^{-1} \Xi\left(X_{i}, \phi\right)\left[\Xi_{n}(\phi)\right]^{-1} g_{n}(\theta), \\
\Xi_{n}(\phi)= & \frac{1}{n} \sum_{i=1}^{n} \Xi\left(X_{i}, \phi\right) .
\end{aligned}
$$

When $\Xi_{n}(\phi)=\Xi\left(X_{i}, \phi\right)=I$, the last term of $m\left(\theta, \Xi_{n}(\phi)\right)$ drops. Note that $m_{i}\left(\theta, \Xi_{n}(\phi)\right)$ does not have to include the centered processes for $G\left(X_{i}\right)$ and $\Xi\left(X_{i}, \phi\right)$ because the FOCs hold evaluated at $\left(\hat{\theta}_{2}, \Omega_{n}\left(\hat{\theta}_{1}\right)\right)$ and $\left(\hat{\theta}_{1}, W_{n}\right)$, respectively.

The doubly corrected variance estimator for the two-step GMM, $\widehat{V}_{d c}\left(\hat{\theta}_{2}\right)$, provides the same order of finite sample correction as the Windmeijer correction, $\widehat{V}_{w}\left(\hat{\theta}_{2}\right)$. The standard error is obtained by taking the diagonal elements of $\sqrt{\widehat{V}_{d c}\left(\hat{\theta}_{2}\right) / n}$.

The doubly corrected variance estimator for the one-step GMM, $\widehat{V}_{d c}\left(\hat{\theta}_{1}\right)$, takes into account for the variations up to the order of $O_{p}\left(n^{-1 / 2}\right)$ in the expansion (19)-(21). This correction is not considered in Windmeijer (2005). The standard error is obtained by taking the diagonal elements of $\sqrt{\widehat{V}_{d c}\left(\hat{\theta}_{1}\right) / n}$.

\section{Stochastic Expansion of GMM Estimators}

In this section, we provide formal stochastic expansions of GMM estimators assuming that the moment condition evaluated at the true value is a local drifting sequence around zero:

$$
E\left[g\left(X_{i n}, \theta_{0}\right)\right]=\frac{\delta}{\sqrt{n}}
$$

for some nonzero $\delta \in \mathbb{R}^{q}$ that depends on $\theta_{0}$. Note that the observations now form a triangular array $\left\{X_{i n}: i=1, \ldots, n, n \in \mathbb{N}\right\}$ because (33) changes with $n$. Under (33) and regularity conditions, $\hat{\theta}_{1}$ and $\hat{\theta}_{2}$ are both consistent for $\theta_{0}$ and by the central limit theorem

$$
\sqrt{n}\left(g_{n}\left(\theta_{0}\right)-E\left[g\left(X_{i n}, \theta_{0}\right)\right]\right)=O_{p}(1) .
$$

This setup is referred to as local misspecification in the literature, e.g., Newey (1985), Otsu (2011), Guggenberger (2012), Conley, Hansen, and Rossi (2012), Andrews, Gentzkow, and Shapiro (2017), Bonhomme and Weidner (2018), and Armstrong and Kolesár (2019). Since $g_{n}\left(\theta_{0}\right)$ is still $O_{p}\left(n^{-1 / 2}\right)$, local misspecification and over-identification bias are essentially equivalent. This setup is useful in distinguishing the effect of the over-identification bias (local misspecification) on the point estimate and on the variance. That is, the local-to-zero device provides a better approximation of the finite sample distribution of the estimator but it should not be taken as the true data-generating process. 
Under correct specification (1), Newey and Smith (2004) provide a thorough analysis on the higher-order bias and variance of the stochastic expansions of GMM and GEL estimators up to the order of $O_{p}\left(n^{-1}\right)$ in the form of

$$
\sqrt{n}\left(\hat{\theta}-\theta_{0}\right)=\psi_{0}+\psi_{1} / \sqrt{n}+\psi_{2} / n+R_{n}
$$

where $\psi_{0}, \psi_{1}, \psi_{2}$ are all $O_{p}(1)$ and $R_{n}=O_{p}\left(n^{-3 / 2}\right)$. Our analysis reveals additional higher-order terms of the order $O_{p}\left(n^{-1 / 2}\right)$ in the RHS of (35) as well as the first order asymptotic bias in the point estimate. Specifically, we derive the exact expressions of $\psi_{0}$ and $\psi_{1}$ for the one-step and the two-step GMM to show that our double correction approximates the (co)variance of some higher-order terms. Deriving the full expression of $\psi_{2}$ is not attempted because the derivation is quite tedious and is not required to show the relationship between the double correction and the higher-order expansion.

Let $g(\theta)=E\left[g\left(X_{i n}, \theta\right)\right]$ and write $g=g\left(\theta_{0}\right)$. Define

$$
\begin{aligned}
\widetilde{g}(\theta) & =\sum_{i=1}^{n}\left(g\left(X_{i n}, \theta\right)-g(\theta)\right) / \sqrt{n} \\
\widetilde{G} & =\sum_{i=1}^{n}\left(G\left(X_{i n}\right)-G\right) / \sqrt{n} \\
\widetilde{W} & =\sum_{i=1}^{n}\left(W\left(X_{i n}\right)-W\right) / \sqrt{n} \\
\widetilde{\Omega}(\theta) & =\sum_{i=1}^{n}\left(g\left(X_{i n}, \theta\right) g\left(X_{i n}, \theta\right)^{\prime}-\Omega(\theta)\right) / \sqrt{n}
\end{aligned}
$$

and write $\widetilde{g}=\widetilde{g}\left(\theta_{0}\right)$ and $\widetilde{\Omega}=\widetilde{\Omega}\left(\theta_{0}\right)$. First we consider the one-step GMM estimator.

Theorem 1. Suppose that (33) holds, $\widetilde{g}=O_{p}(1), \widetilde{G}=O_{p}(1), \widetilde{W}=O_{p}(1)$, the FOC of the one-step GMM holds with probability approaching one (w.p.a. 1), $G$ is full column rank, and $W>0$. In addition, suppose that the second moment of $g\left(X_{i n}, \theta_{0}\right), G\left(X_{i n}\right)$, and $W\left(X_{i n}\right)$ exist and are finite. Then, the one-step GMM estimator has the following expansion w.p.a.1.

$$
\sqrt{n}\left(\hat{\theta}_{1}-\theta_{0}\right)=\eta_{W}+\widetilde{\psi}_{W, 0}+\frac{1}{\sqrt{n}}\left(\widetilde{\psi}_{W, 1}+\widetilde{q}_{W}+\widetilde{B}_{W}\left(\eta_{W}+\widetilde{\psi}_{W, 0}\right)\right)+O_{p}\left(\frac{1}{n}\right)
$$


where

$$
\begin{aligned}
\eta_{W} & =-\left(G^{\prime} W^{-1} G\right)^{-1} G^{\prime} W^{-1} \delta \\
\widetilde{\psi}_{W, 0} & =-\left(G^{\prime} W^{-1} G\right)^{-1} G^{\prime} W^{-1} \widetilde{g} \\
\widetilde{\psi}_{W, 1} & =-\left(G^{\prime} W^{-1} G\right)^{-1}\left(\widetilde{G}^{\prime} W^{-1} \delta-G^{\prime} W^{-1} \widetilde{W} W^{-1} \delta\right), \\
\widetilde{q}_{W} & =-\left(G^{\prime} W^{-1} G\right)^{-1}\left(\widetilde{G} W^{-1} \widetilde{g}-G^{\prime} W^{-1} \widetilde{W} W^{-1} \widetilde{g}\right) \\
\widetilde{B}_{W} & =-\left(G^{\prime} W^{-1} G\right)^{-1}\left(\widetilde{G}^{\prime} W^{-1} G-G^{\prime} W^{-1} \widetilde{W} W^{-1} G+G^{\prime} W^{-1} \widetilde{G}\right) .
\end{aligned}
$$

The first term in the expansion of Theorem $1, \eta_{W}$, is the constant bias due to local misspecification. It shifts the mean of the distribution but does not alter the variance. This bias cannot be consistently estimated from the data in general. Conley, Hansen, and Rossi (2012) impose a prior distribution on $\delta$ and Armstrong and Kolesár (2019) set a pre-specified bound on $\delta$ to construct bias-corrected confidence intervals.

The conventional variance estimator estimates the variance of $\widetilde{\psi}_{W, 0}$. The doubly corrected variance estimator (30) estimates the variance of $\widetilde{\psi}_{W, 0}+\widetilde{\psi}_{W, 1} / \sqrt{n}$. Since the variance of $\widetilde{\psi}_{W, 1}$ increases with $\delta$, the double correction would be more effective with a larger $\delta$. This does not necessarily imply potentially larger bias in the point estimate, however. The magnitude of change in $\eta_{W}$ and in the variance due to $\delta$ depends on the data matrices. For example, Andrews, Gentzkow, and Shapiro (2017) use $\eta_{W}$ with a given $\delta$ to check how sensitive the estimator is. So if the estimator is not sensitive (to local violation of the moment condition), then $\eta_{W}$ does not change much while the variance may be significantly affected. Table 1 shows an example that the bias in the point estimate does not increase with $\delta$ while the standard deviation of the estimator increases substantially $(n=50)$.

On the other hand, the double correction omits some terms of the same order, $\left(\widetilde{q}_{W}+\widetilde{B}_{W}\left(\eta_{W}+\right.\right.$ $\left.\left.\widetilde{\psi}_{W, 0}\right)\right) / \sqrt{n}$. We discuss these terms one by one. First, the variance of $\left(\widetilde{q}_{W}+\widetilde{B}_{W} \widetilde{\psi}_{W, 0}\right) / \sqrt{n}$ does not increase with $\delta$ because $\widetilde{g}$ is a centered process. Second, $\operatorname{Var}\left(\widetilde{B}_{W} \eta_{W} / \sqrt{n}\right)$ increases with $\delta$ but its sample analogue is zero because the sample analogue of $\eta_{W}$ is zero (FOC). So the (co)variance of this term cannot be estimated.

An estimator of the higher-order variance up to $O_{p}\left(n^{-1}\right)$ of Newey and Smith (2004) would include the variance of $\left(\widetilde{q}_{W}+\widetilde{B}_{W}\left(\eta_{W}+\widetilde{\psi}_{W, 0}\right)\right) / \sqrt{n}$ and the covariance between $\widetilde{\psi}_{W, 0}$ and $\left(\widetilde{q}_{W}+\right.$ $\left.\widetilde{B}_{W}\left(\eta_{W}+\widetilde{\psi}_{W, 0}\right)\right) / \sqrt{n}+\widetilde{\psi}_{W, 2} / n$ where $\widetilde{\psi}_{W, 2}$ is the higher-order term corresponding $\psi_{2}$ in (35). Since these terms are not taken into account, the doubly corrected variance estimator is not an estimator of the higher-order variance of Newey and Smith (2004). Although the higher-order variance up to $O_{p}\left(n^{-1}\right)$ could be estimated by further expanding (36) up to the remainder term of the order $O_{p}\left(n^{-3 / 2}\right)$ the resulting estimator would include many terms to be estimated which would not be practical. Thus, the doubly corrected variance estimator can be viewed as a convenient alternative to the higher-order variance estimator.

Next we consider the two-step GMM estimator. 
Theorem 2. Suppose that the assumptions of Theorem 1 hold. In addition, $\widetilde{\Omega}=O_{p}(1)$, the FOC of the two-step GMM holds w.p.a.1, $\Omega>0$, and the fourth moment of $g\left(X_{i n}, \theta_{0}\right)$ exists and is finite. Then the two-step GMM estimator has the following expansion w.p.a.1.

$$
\begin{aligned}
& \sqrt{n}\left(\hat{\theta}_{2}-\theta_{0}\right) \\
= & {\left[\eta_{\Omega}+\frac{1}{\sqrt{n}}\left(\left(D+H_{\eta_{\Omega}}\right) \eta_{W}\right)\right] } \\
& +\widetilde{\psi}_{\Omega, 0}+\frac{1}{\sqrt{n}}\left(\widetilde{\psi}_{\Omega, 1}+\left(D+\widetilde{C}+H_{\eta_{\Omega}}+H_{\widetilde{\psi}_{\Omega, 0}}\right) \widetilde{\psi}_{W, 0}+\widetilde{q}_{\Omega}+\widetilde{B}_{\Omega}\left(\eta_{\Omega}+\widetilde{\psi}_{\Omega, 0}\right)+\left(\widetilde{C}+H_{\widetilde{\psi}_{\Omega, 0}}\right) \eta_{W}\right) \\
& +\frac{1}{n} D \widetilde{\psi}_{W, 1}+O_{p}\left(\frac{1}{n}\right)
\end{aligned}
$$

where

$$
\begin{aligned}
\eta_{\Omega} & =-\left(G^{\prime} \Omega^{-1} G\right)^{-1} G^{\prime} \Omega^{-1} \delta, \\
\widetilde{\psi}_{\Omega, 0} & =-\left(G^{\prime} \Omega^{-1} G\right)^{-1} G^{\prime} \Omega^{-1} \widetilde{g} \\
\widetilde{\psi}_{\Omega, 1} & =-\left(G^{\prime} \Omega^{-1} G\right)^{-1}\left(\widetilde{G}^{\prime} \Omega^{-1} \delta-G^{\prime} \Omega^{-1} \widetilde{\Omega} \Omega^{-1} \delta\right), \\
\widetilde{q}_{\Omega} & =-\left(G^{\prime} \Omega^{-1} G\right)^{-1}\left(\widetilde{G}^{\prime} \Omega^{-1} \widetilde{g}-G^{\prime} \Omega^{-1} \widetilde{\Omega} \Omega^{-1} \widetilde{g}\right), \\
\widetilde{B}_{\Omega} & =-\left(G^{\prime} \Omega^{-1} G\right)^{-1}\left(\widetilde{G}^{\prime} \Omega^{-1} G-G^{\prime} \Omega^{-1} \widetilde{\Omega} \Omega^{-1} G+G^{\prime} \Omega^{-1} \widetilde{G}\right), \\
D[., j] & =\left.\left(G^{\prime} \Omega^{-1} G\right)^{-1} G^{\prime} \Omega^{-1} \frac{\partial \Omega(\theta)}{\partial \theta_{[j]}}\right|_{\theta=\theta_{0}} \Omega^{-1} \delta \\
\widetilde{C}[., j] & =\left.\left(G^{\prime} \Omega^{-1} G\right)^{-1} G^{\prime} \Omega^{-1} \frac{\partial \Omega(\theta)}{\partial \theta_{[j]}}\right|_{\theta=\theta_{0}} \Omega^{-1} \widetilde{g}, \\
H_{v}[., j] & =\left.\left(G^{\prime} \Omega^{-1} G\right)^{-1} G^{\prime} \Omega^{-1} \frac{\partial \Omega(\theta)}{\partial \theta_{[j]}}\right|_{\theta=\theta_{0}} \Omega^{-1} G v .
\end{aligned}
$$

The terms in the square brackets in the expansion, $\eta_{\Omega}+\left(D+H_{\eta_{\Omega}}\right) \eta_{W} / \sqrt{n}$ is the constant bias due to local misspecification which cannot be consistently estimated in general. It only shifts the mean of the distribution but does not affect the variance.

The conventional, the Windmeijer correction, and the doubly corrected variance estimators estimate the variance of

$$
\begin{aligned}
\text { conventional: } & \widetilde{\psi}_{\Omega, 0}, \\
\text { Windmeijer: } & \widetilde{\psi}_{\Omega, 0}+\frac{D \widetilde{\psi}_{W, 0}}{\sqrt{n}}, \\
\text { double correction: } & \widetilde{\psi}_{\Omega, 0}+\frac{\widetilde{\psi}_{\Omega, 1}+D \widetilde{\psi}_{W, 0}}{\sqrt{n}}+\frac{D \widetilde{\psi}_{W, 1}}{n},
\end{aligned}
$$

respectively. Similar to the one-step GMM expansion, the double correction estimates the variance of the higher-order terms that increase with $\delta: D, \widetilde{\psi}_{\Omega, 1}$, and $\widetilde{\psi}_{W, 1}$. In contrast, some (or all) of 
these terms are omitted in the Windmeijer correction (or the conventional variance estimator). This implies that those variance estimators tend to differ more with a larger $\delta$, which is also supported by the simulation experiment in Section 8 . Note that a larger $\delta$ does not necessarily imply larger bias in the point estimate via $\eta_{\Omega}$ and $\eta_{W}$. For example, Table 1 shows that the bias in the two-step GMM point estimate decreases with $\delta$ but the three variance estimators differ substantially for a large $\delta$.

Including $\left(\widetilde{\psi}_{\Omega, 1}+D \widetilde{\psi}_{W, 0}\right) / \sqrt{n}+D \widetilde{\psi}_{W, 1} / n$ in the double correction formula is also important to get robustness to global (fixed) misspecification, where $\delta=O(\sqrt{n})$, because these terms become $O_{p}(1)$. In this case, the expansion should be centered around the pseudo-true value which is defined as the unique minimizer of the GMM criterion and $\eta_{W}$ and $\eta_{\Omega}$ become zero because of the population FOC. We show that the doubly corrected variance estimator is misspecification-robust in the next section.

By a similar argument with the one-step GMM, both the doubly corrected and the Windmeijer corrected variance estimators are not the higher-order variance estimator of Newey and Smith (2004) because some higher-order terms that constitute the higher-order variance up to $O_{p}\left(n^{-1}\right)$ are omitted in both corrections.

Remark (Edgeworth expansion) The improved approximation to the finite sample variance of the GMM estimators by the double correction is different from the higher-order refinement via the Edgeworth expansion which expands the finite sample distribution function of the (standardized) test statistic. Using analytical expansions (Rothenberg, 1984; Hansen, 2006; Kundhi and Rilstone, 2013) or the bootstrap (Hall, 1992; Hall and Horowitz, 1996; Andrews, 2002; Lee, 2014, 2016), a critical value can be obtained which gives a smaller error in the size of the test or in the coverage probability of the confidence interval.

\section{Robustness to Misspecification}

Both the Windmeijer corrected and the conventional variance estimators, $\widehat{V}_{w}\left(\hat{\theta}_{2}\right)$ and $\widetilde{V}\left(\hat{\theta}_{2}\right)$, are consistent for the asymptotic variance of $\sqrt{n}\left(\hat{\theta}_{2}-\theta_{0}\right)$ under correct specification, $E\left[g\left(X_{i}, \theta_{0}\right)\right]=0$. In words, correct specification means that an over-identified model exactly holds at a unique parameter value $\theta_{0}$, but this may be too restrictive in reality. Indeed, the sample moment condition model does not hold for any finite sample size $n$ almost surely if the model is over-identified, i.e., $g_{n}(\hat{\theta}) \neq 0$. Thus, it is reasonable to view the assumed moment condition model as the best approximating model and to allow for possible misspecification.

Under (global) misspecification, which is defined as

$$
E\left[g\left(X_{i}, \theta\right)\right]=\delta(\theta) \neq 0, \forall \theta \in \Theta
$$

where $\delta(\theta)$ is a vector of constants and $\Theta$ is a compact parameter space, the GMM estimator is consistent for the pseudo-true value, which is defined as the unique minimizer of the population 
GMM criterion given the weight matrix (Hall and Inoue, 2003). Thus, the pseudo-true values of the one-step and two-step GMM may differ from each other. In addition, the asymptotic variance has more terms that are assumed away under correct specification. Thus, the conventional variance estimators are no longer consistent under misspecification. Lee (2014) proposes variance estimators for the one-step and two-step GMM under misspecification. Hansen and Lee (2019) propose a similar robust variance estimator for the iterated GMM. These variance estimators are shown to be consistent regardless of misspecification and they are referred to as the misspecification-robust variance estimator, hereinafter.

Since the misspecification-robust variance estimators contain additional terms which are not present in the conventional variance estimator, it has been generally conjectured less accurate than the conventional variance estimator under correct specification. We show that this conjecture is not true by showing that the doubly corrected variance estimator $\widehat{V}_{d c}\left(\hat{\theta}_{2}\right)$ is the misspecification-robust variance estimator.

The equivalence holds for the following reasons. First, the Windmeijer correction that accounts for the effect of $\hat{\theta}_{1}$ in the weight matrix corrects for the pseudo-true value of the one-step GMM being different from that of the two-step GMM. Our double correction corrects for this bias too. Second, the double correction corrects for the over-identification bias which becomes asymptotically non-negligible misspecification bias. Overall, the double correction makes the expansions (23)-(27) robust to misspecification.

To formally show that $\widehat{V}_{d c}\left(\hat{\theta}_{2}\right)$ is consistent for the asymptotic variance under misspecification, we first make some definitions. Define the one-step and two-step GMM (pseudo-)true values as

$$
\begin{aligned}
& \theta_{1}=\underset{\theta}{\arg \min } E\left[g\left(X_{i}, \theta\right)\right]^{\prime} W^{-1} E\left[g\left(X_{i}, \theta\right)\right], \\
& \theta_{2}=\underset{\theta}{\arg \min } E\left[g\left(X_{i}, \theta\right)\right]^{\prime}\left[\Omega\left(\theta_{1}\right)\right]^{-1} E\left[g\left(X_{i}, \theta\right)\right] .
\end{aligned}
$$

In general $\theta_{1} \neq \theta_{2}$ but $\theta_{1}=\theta_{2}=\theta_{0}$ under correct specification. Write $g_{j}=E\left[g\left(X_{i}, \theta_{j}\right)\right]$ and $\Omega_{j}=\Omega\left(\theta_{j}\right)$ for $j=1,2$. (Global) misspecification implies that $g_{n}\left(\theta_{j}\right)=O_{p}(1)$ for $j=1,2$. Sufficient conditions for consistency and asymptotic normality under misspecification for the onestep and two-step GMM is given in Hall and Inoue (2003) for stationary data and for the one-step and iterated GMM is given in B. Hansen and Lee (2019) for clustered data.

Take the FOC of the two-step GMM. We can use the expansions (6)-(8) by letting $\theta_{1} \neq \theta_{2}$. Then (9) can be written as

$$
\begin{aligned}
\sqrt{n}\left(\hat{\theta}_{2}-\theta_{2}\right)= & -\left\{G_{n}^{\prime}\left[\Omega_{n}\left(\theta_{1}\right)\right]^{-1} G_{n}\right\}^{-1} G_{n}^{\prime}\left[\Omega_{n}\left(\theta_{1}\right)\right]^{-1} \sqrt{n} g_{n}\left(\theta_{2}\right) \\
& +D_{n}^{*} \sqrt{n}\left(\hat{\theta}_{1}-\theta_{1}\right)+O_{p}\left(n^{-1 / 2}\left\|g_{n}\left(\theta_{2}\right)\right\|\right) \\
= & \sqrt{n}\left(\tilde{\theta}_{2}^{*}-\theta_{2}\right)+D_{n}^{*} \sqrt{n}\left(\hat{\theta}_{1}-\theta_{1}\right)+O_{p}\left(n^{-1 / 2}\left\|g_{n}\left(\theta_{2}\right)\right\|\right),
\end{aligned}
$$

where $\tilde{\theta}_{2}^{*}$ is defined as

$$
\tilde{\theta}_{2}^{*}=\arg \min _{\theta \in \Theta} g_{n}(\theta)^{\prime}\left[\Omega_{n}\left(\theta_{1}\right)\right]^{-1} g_{n}(\theta)
$$


and

$$
\begin{aligned}
& D_{n}^{*}=F_{1 n}^{*}+F_{2 n}^{*}, \\
& F_{1 n}^{*}=-\left.\frac{\partial\left\{G_{n}^{\prime}\left[\Omega_{n}(\theta)\right]^{-1} G_{n}\right\}^{-1}}{\partial \theta^{\prime}}\right|_{\theta=\theta_{1}} G_{n}^{\prime}\left[\Omega_{n}\left(\theta_{1}\right)\right]^{-1} g_{n}\left(\theta_{2}\right), \\
& F_{2 n}^{*}=-\left.\left\{G_{n}^{\prime}\left[\Omega_{n}\left(\theta_{1}\right)\right]^{-1} G_{n}\right\}^{-1} \frac{\partial G_{n}^{\prime}\left[\Omega_{n}(\theta)\right]^{-1} g_{n}\left(\theta_{2}\right)}{\partial \theta^{\prime}}\right|_{\theta=\theta_{1}} .
\end{aligned}
$$

Since $D_{n}^{*}=O_{p}\left(\left\|g_{n}\left(\theta_{2}\right)\right\|\right)$, the order of finite sample correction depends on the degree of misspecification, from being $O_{p}\left(n^{-1 / 2}\right)$ under correct specification to $O_{p}(1)$ under (global) misspecification. Note that both $\sqrt{n}\left(\tilde{\theta}_{2}^{*}-\theta_{2}\right)$ and $D_{n}^{*} \sqrt{n}\left(\hat{\theta}_{1}-\theta_{1}\right)$ in (53) are $O_{p}(1)$ under misspecification and this will alter the first-order asymptotic variance.

Using the fact that $G^{\prime} \Omega_{j}^{-1} g_{j}=0$ for $j=1,2$, the FOC of the infeasible two-step GMM (54) can be expanded as

$$
\begin{aligned}
\sqrt{n}\left(\tilde{\theta}_{2}^{*}-\theta_{2}\right)= & -\left\{G_{n}^{\prime}\left[\Omega_{n}\left(\theta_{1}\right)\right]^{-1} G_{n}\right\}^{-1}\left\{G_{n}^{\prime}\left[\Omega_{n}\left(\theta_{1}\right)\right]^{-1} \sqrt{n}\left(g_{n}\left(\theta_{2}\right)-g_{2}\right)\right. \\
& \left.+\sqrt{n}\left(G_{n}-G\right)^{\prime}\left[\Omega_{n}\left(\theta_{1}\right)\right]^{-1} g_{2}-G^{\prime} \Omega_{1}^{-1} \sqrt{n}\left(\Omega_{n}\left(\theta_{1}\right)-\Omega_{1}\right)\left[\Omega_{n}\left(\theta_{1}\right)\right]^{-1} g_{2}\right\} .
\end{aligned}
$$

The FOC of the one-step GMM can be expanded similarly:

$$
\begin{aligned}
\sqrt{n}\left(\hat{\theta}_{1}-\theta_{1}\right)= & -\left\{G_{n}^{\prime} W_{n}^{-1} G_{n}\right\}^{-1}\left[G_{n}^{\prime} W_{n}^{-1} \sqrt{n}\left(g_{n}\left(\theta_{1}\right)-g_{1}\right)\right. \\
& \left.+\sqrt{n}\left(G_{n}-G\right)^{\prime} W_{n}^{-1} g_{1}-G^{\prime} W^{-1} \sqrt{n}\left(W_{n}-W\right) W_{n}^{-1} g_{1}\right] .
\end{aligned}
$$

The expansions (55) and (56) are misspecification-robust versions of (7) and (8), allowing for different probability limits of the one-step and two-step GMM estimators and taking into account for the misspecification (over-identification) bias.

The following theorem shows that the doubly corrected variance estimators of the one-step and two-step linear GMM are consistent for the asymptotic variance matrices under misspecification. The proof is given in the appendix.

Theorem 3. Suppose that (50) holds, $G_{n}-G=O_{p}\left(n^{-1 / 2}\right), W_{n}-W=O_{p}\left(n^{-1 / 2}\right), \Omega_{n}\left(\theta_{j}\right)-\Omega_{j}=$ $O_{p}\left(n^{-1 / 2}\right), \hat{\theta}_{j}-\theta_{j}=O_{p}\left(n^{-1 / 2}\right)$ for $j=1,2, W$ and $\Omega_{1}$ are nonsingular, and $G$ is full column rank. Then, as $n \rightarrow \infty$, for $j=1,2$,

$$
\widehat{V}_{d c}\left(\hat{\theta}_{j}\right)^{-1 / 2} \sqrt{n}\left(\hat{\theta}_{j}-\theta_{j}\right) \stackrel{d}{\rightarrow} N\left(0, I_{k}\right)
$$

Theorem 3 also implies that the bootstrap $t$ test and confidence intervals (CIs) based on the GMM $t$ statistic studentized with the doubly corrected standard error automatically achieve higherorder refinements over the asymptotic $t$ test and CIs regardless of misspecification (Lee, 2014). In contrast, the bootstrap $t$ test and CIs based on the GMM $t$ statistic studentized with the conven- 
tional or the Windmeijer standard error requires an additional recentering procedure in resampling to correct for the over-identification bias to achieve higher-order refinements. Furthermore, the conventional bootstrap procedure with recentering is not valid under misspecification. Thus, our doubly corrected variance estimator formula provides a very convenient way to get more accurate but also robust bootstrap tests and CIs.

Remark (Weight matrix) All the results so far hold if we replace $\Omega_{n}(\theta)$ with the centered weight matrix

$$
\Omega_{n}^{c}(\theta)=\frac{1}{n} \sum_{i=1}^{n}\left(g\left(X_{i}, \theta\right)-g_{n}(\theta)\right)\left(g\left(X_{i}, \theta\right)-g_{n}(\theta)\right)^{\prime} .
$$

The matrices $D_{n}$ and $\widehat{D}_{n}$ would need to be modified accordingly. The centered weight matrix is consistent for the asymptotic variance matrix of the moment equation under misspecification. B. Hansen (2019) recommends using the centered weight matrix for this reason. Hall (2000) shows that the GMM over-identification test statistic with a centered heteroskedasticity-and-autocorrelationconsistent (HAC) weight matrix leads to more powerful tests in the time series setting.

\section{Iterated GMM and Continuously Updating GMM}

Both the Windmeijer and our double correction correct for the extra variation due to the weight matrix being evaluated at an estimate rather than the true value. A natural question is whether similar finite sample corrections can be obtained for other GMM estimators, namely the iterated GMM of B. Hansen and Lee (2019) and the continuously-updating (CU) GMM of L. Hansen, Heaton, and Yaron (1996). We show that the answer is yes for the iterated GMM and also show that the double correction formula is the same as the misspecification-robust formula. For the CU GMM, the answer is negative.

Assume correct specification. The iterated GMM estimator is obtained by iterating the two-step efficient GMM estimator until convergence. By iteration the dependence of the final estimator on the previous step estimators disappears. The FOC is given by

$$
0=G_{n}^{\prime}\left[\Omega_{n}(\hat{\theta})\right]^{-1} g_{n}(\hat{\theta})
$$

where $\hat{\theta}$ is the iterated GMM. Assume that $g_{n}\left(\theta_{0}\right)=O_{p}\left(n^{-1 / 2}\right)$ and $\hat{\theta}-\theta_{0}=O_{p}\left(n^{-1 / 2}\right)$ whose sufficient conditions are provided in Hansen and Lee (2019). By applying the first-order Taylor expansion around $\theta_{0}$ to $g_{n}(\hat{\theta})$ and $\Omega_{n}(\hat{\theta})$ sequentially

$$
\sqrt{n}\left(\hat{\theta}-\theta_{0}\right)=-\left\{G_{n}^{\prime}\left[\Omega_{n}\left(\theta_{0}\right)\right]^{-1} G_{n}\right\}^{-1} G_{n}^{\prime}\left[\Omega_{n}\left(\theta_{0}\right)\right]^{-1} \sqrt{n} g_{n}\left(\theta_{0}\right)+D_{n} \sqrt{n}\left(\hat{\theta}-\theta_{0}\right)+O_{p}\left(n^{-1}\right)
$$

and thus

$$
\sqrt{n}\left(\hat{\theta}-\theta_{0}\right)=-\left\{G_{n}^{\prime}\left[\Omega_{n}\left(\theta_{0}\right)\right]^{-1} G_{n}\left(I_{k}-D_{n}\right)\right\}^{-1} G_{n}^{\prime}\left[\Omega_{n}\left(\theta_{0}\right)\right]^{-1} \sqrt{n} g_{n}\left(\theta_{0}\right)+O_{p}\left(n^{-1}\right) .
$$


Windmeijer (2000) proposes a finite sample corrected variance estimator based on the expansion (60). We proceed one additional step. By further expanding to take into account for the overidentification bias, we have

$$
\begin{aligned}
\sqrt{n}\left(\hat{\theta}-\theta_{0}\right) & =-\left\{G_{n}^{\prime}\left[\Omega_{n}\left(\theta_{0}\right)\right]^{-1} G_{n}\left(I_{k}-D_{n}\right)\right\}^{-1}\left[G^{\prime} \Omega^{-1} \sqrt{n} g_{n}\left(\theta_{0}\right)\right. \\
& \left.+\sqrt{n}\left(G_{n}-G\right)^{\prime} \Omega^{-1} g_{n}\left(\theta_{0}\right)-G^{\prime} \Omega^{-1} \sqrt{n}\left(\Omega_{n}\left(\theta_{0}\right)-\Omega\right) \Omega^{-1} g_{n}\left(\theta_{0}\right)\right]+O_{p}\left(n^{-1}\right) .
\end{aligned}
$$

Since the remainder term is $O_{p}\left(n^{-1}\right)$, by estimating the variance of the terms in (61)-(62) up to $O_{p}\left(n^{-1 / 2}\right)$ we can get the same order of finite sample correction with the doubly corrected two-step GMM variance estimator.

The doubly corrected variance estimator for the iterated GMM is

$$
\widehat{V}_{d c}(\hat{\theta})=\left\{G_{n}^{\prime}\left[\Omega_{n}(\hat{\theta})\right]^{-1} G_{n}\left(I_{k}-\widehat{D}_{n}\right)\right\}^{-1} \Sigma_{n}\left(\hat{\theta}, \Omega_{n}(\hat{\theta})\right)\left\{G_{n}^{\prime}\left[\Omega_{n}(\hat{\theta})\right]^{-1} G_{n}\left(I_{k}-\widehat{D}_{n}\right)\right\}^{-1 \prime},
$$

where $\Sigma_{n}\left(\hat{\theta}, \Omega_{n}(\hat{\theta})\right)$ is defined in (32) and $\widehat{D}_{n}$ is evaluated at $\hat{\theta}$. Not surprisingly, this formula is identical to the misspecification-robust variance estimator for the iterated GMM of Hansen and Lee (2019). The finite sample corrected formula suggested by Windmeijer (2000) is

$$
\widehat{V}_{w}(\hat{\theta})=\left(I_{k}-\widehat{D}_{n}\right)^{-1}\left(G_{n}^{\prime}\left[\Omega_{n}(\hat{\theta})\right]^{-1} G_{n}\right)^{-1}\left(I_{k}-\widehat{D}_{n}\right)^{-1^{\prime}}
$$

On the other hand, a similar finite sample correction may not be obtained for the CU GMM. Windmeijer (2005) showed that if the derivative of the moment function is a function of the parameter, then the proposed formula would not necessarily give finite sample corrections. The same argument applied to CU GMM. Let $\hat{\theta}$ be the CU GMM estimator. For simplicity, let $k=1$ so that $\theta$ is scalar. The FOC is

$$
0=\left(G_{n}-\left.\frac{1}{2} g_{n}(\hat{\theta})^{\prime}\left[\Omega_{n}(\hat{\theta})\right]^{-1} \frac{\partial \Omega_{n}(\theta)}{\partial \theta}\right|_{\theta=\hat{\theta}}\right)^{\prime}\left[\Omega_{n}(\hat{\theta})\right]^{-1} g_{n}(\hat{\theta})
$$

This shows that even when the moment function is linear, the effective derivative that forms the FOC still depends on the parameter. Thus, the misspecification-robust variance formula for CU GMM does not necessarily provide a finite sample correction under correct specification. Since GEL estimators have similar non-linear FOC even with linear moment functions, we expect similar conclusions. 


\section{Examples}

\subsection{Cross-sectional IV}

Consider the linear IV model $y_{i}=X_{i}^{\prime} \theta+e_{i}$ with the moment conditions $E\left[Z_{i} e_{i}\right]=0$. The two-stage least squares (2SLS) estimator is given by

$$
\hat{\theta}_{1}=\left(X^{\prime} Z\left(Z^{\prime} Z\right)^{-1} Z^{\prime} X\right)^{-1} X^{\prime} Z\left(Z^{\prime} Z\right)^{-1} Z^{\prime} Y
$$

where $Y=\left[y_{1}, \cdots, y_{n}\right]^{\prime}, X=\left[X_{1}, \cdots, X_{n}\right]^{\prime}$, and $Z=\left[Z_{1}, \cdots, Z_{n}\right]^{\prime}$ are $n \times 1, n \times k$, and $n \times q$ data matrices. Using the 2SLS as the preliminary estimator, the two-step efficient GMM estimator is given by

$$
\hat{\theta}_{2}=\left(X^{\prime} Z \widehat{\Omega}_{1}^{-1} Z^{\prime} X\right)^{-1} X^{\prime} Z \widehat{\Omega}_{1}^{-1} Z^{\prime} Y
$$

where

$$
\begin{aligned}
& \widehat{\Omega}_{1}=\frac{1}{n} \sum_{i=1}^{n} Z_{i} Z_{i}^{\prime} \hat{e}_{1 i}^{2}, \\
& \hat{e}_{1 i}=y_{i}-X_{i}^{\prime} \hat{\theta}_{1} .
\end{aligned}
$$

Also define $\hat{e}_{2 i}=y_{i}-X_{i}^{\prime} \hat{\theta}_{2}$ and the $n \times 1$ residual vector $\hat{e}_{j}=Y-X \hat{\theta}_{j}$ for $j=1,2$.

The doubly corrected variance estimators of the 2SLS and two-step GMM are

$$
\begin{aligned}
& \widehat{V}_{d c}\left(\hat{\theta}_{1}\right)=\left(\frac{1}{n} X^{\prime} Z\left(Z^{\prime} Z\right)^{-1} Z^{\prime} X\right)^{-1} \frac{1}{n} \sum_{i=1}^{n} \hat{m}_{1 i} \hat{m}_{1 i}^{\prime}\left(\frac{1}{n} X^{\prime} Z\left(Z^{\prime} Z\right)^{-1} Z^{\prime} X\right)^{-1}, \\
& \widehat{V}_{d c}\left(\hat{\theta}_{2}\right)=\widehat{V}\left(\hat{\theta}_{2}\right)+\widehat{D}_{n} \widehat{C}\left(\hat{\theta}_{1}, \hat{\theta}_{2}\right)+\widehat{C}\left(\hat{\theta}_{1}, \hat{\theta}_{2}\right)^{\prime} \widehat{D}_{n}^{\prime}+\widehat{D}_{n} \widehat{V}_{d c}\left(\hat{\theta}_{1}\right) \widehat{D}_{n}^{\prime}
\end{aligned}
$$

where

$$
\begin{aligned}
\widehat{V}\left(\hat{\theta}_{2}\right) & =\left(\frac{1}{n^{2}} X^{\prime} Z \widehat{\Omega}_{1}^{-1} Z^{\prime} X\right)^{-1}\left(\frac{1}{n} \sum_{i=1}^{n} \hat{m}_{2 i} \hat{m}_{2 i}^{\prime}\right)\left(\frac{1}{n^{2}} X^{\prime} Z \widehat{\Omega}_{1}^{-1} Z^{\prime} X\right)^{-1}, \\
\widehat{C}\left(\hat{\theta}_{1}, \hat{\theta}_{2}\right) & =\left(\frac{1}{n} X^{\prime} Z\left(Z^{\prime} Z\right)^{-1} Z^{\prime} X\right)^{-1}\left(\frac{1}{n} \sum_{i=1}^{n} \hat{m}_{1 i} \hat{m}_{2 i}^{\prime}\right)\left(\frac{1}{n^{2}} X^{\prime} Z \widehat{\Omega}_{1}^{-1} Z^{\prime} X\right)^{-1}, \\
\widehat{D}_{n} & =\frac{2}{n}\left(X^{\prime} Z \widehat{\Omega}_{1}^{-1} Z^{\prime} X\right)^{-1} X^{\prime} Z \widehat{\Omega}_{1}^{-1} \sum_{i=1}^{n} Z_{i}\left(\hat{e}_{1 i} Z_{i}^{\prime} \widehat{\Omega}_{1}^{-1} Z^{\prime} \hat{e}_{2}\right) X_{i}^{\prime}, \\
\hat{m}_{1 i} & =X^{\prime} Z\left(Z^{\prime} Z\right)^{-1} Z_{i} \hat{e}_{1 i}+X_{i} Z_{i}^{\prime}\left(Z^{\prime} Z\right)^{-1} Z^{\prime} \hat{e}_{1}-X^{\prime} Z\left(Z^{\prime} Z\right)^{-1} Z_{i} Z_{i}^{\prime}\left(Z^{\prime} Z\right)^{-1} Z^{\prime} \hat{e}_{1}, \\
\hat{m}_{2 i} & =\frac{1}{n} X^{\prime} Z \widehat{\Omega}_{1}^{-1} Z_{i} \hat{e}_{2 i}+\frac{1}{n} X_{i} Z_{i}^{\prime} \widehat{\Omega}_{1}^{-1} Z^{\prime} \hat{e}_{2}-\frac{1}{n^{2}} X^{\prime} Z \widehat{\Omega}_{1}^{-1} Z_{i} Z_{i}^{\prime} \hat{e}_{1 i}^{2} \widehat{\Omega}_{1}^{-1} Z^{\prime} \hat{e}_{2} .
\end{aligned}
$$

It is worth observing that the doubly corrected variance estimator $\widehat{V}_{d c}\left(\hat{\theta}_{2}\right)$ reduces to the Windmeijer corrected one $\widehat{V}_{w}\left(\hat{\theta}_{2}\right)$ if (i) the last two terms in $\hat{m}_{2 i}$ and $\hat{m}_{1 i}$ are ignored and (ii) $\hat{e}_{1 i}$ replaces $\hat{e}_{2 i}$ in $\hat{m}_{2 i}$. By (i) and (ii), the variance estimators $\widehat{V}\left(\hat{\theta}_{2}\right)$ and $\widehat{V}_{d c}\left(\hat{\theta}_{1}\right)$ reduce to conventional ones 
$\widetilde{V}\left(\hat{\theta}_{2}\right)$ and $\widetilde{V}\left(\hat{\theta}_{1}\right)$, and $\widehat{C}\left(\hat{\theta}_{1}, \hat{\theta}_{2}\right)$ becomes $\widetilde{V}\left(\hat{\theta}_{2}\right)$. In general, however, $\widehat{V}_{d c}\left(\hat{\theta}_{2}\right) \neq \widehat{V}_{w}\left(\hat{\theta}_{2}\right)$ because $Z^{\prime} \hat{e}_{j} \neq 0$ for $j=1,2$, so the last two terms of $\hat{m}_{j i}$ are non-zero. Furthermore, it is critical (and reasonable) to use $\hat{e}_{2 i}$ in $\hat{m}_{2 i}$ to get robustness under misspecification.

The iterated GMM estimator is obtained as follows. Let $\hat{\theta}_{0}$ be any initial value. The $s$-step GMM estimator for $s \geq 1$ is given by

$$
\hat{\theta}_{s}=\left(X^{\prime} Z \widehat{\Omega}_{s-1}^{-1} Z^{\prime} X\right)^{-1} X^{\prime} Z \widehat{\Omega}_{s-1}^{-1} Z^{\prime} Y,
$$

where

$$
\widehat{\Omega}_{s-1}=\frac{1}{n} \sum_{i=1}^{n} Z_{i} Z_{i}^{\prime}\left(y_{i}-X_{i}^{\prime} \hat{\theta}_{s-1}\right)^{2} .
$$

We iterate the $s$-step GMM estimator until convergence given a preset tolerance, e.g. $\left\|\hat{\theta}_{s}-\hat{\theta}_{s-1}\right\|<$ $10^{-5}$ to obtain the iterated GMM estimator $\hat{\theta}$. The residuals are $\hat{e}_{i}=y_{i}-X_{i}^{\prime} \hat{\theta}$. Also let $\hat{e}=Y-X \hat{\theta}$ be the $n \times 1$ residual vector.

The doubly corrected variance estimator is

$$
\begin{aligned}
\widehat{V}_{d c}(\hat{\theta}) & =\widehat{H}^{-1}\left(\frac{1}{n} \sum_{i=1}^{n} \hat{m}_{i} \hat{m}_{i}^{\prime}\right) \widehat{H}^{-1 \prime}, \\
\widehat{H} & =\frac{1}{n^{2}} X^{\prime} Z \widehat{\Omega}^{-1} Z^{\prime} X-\frac{2}{n^{3}} X^{\prime} Z \widehat{\Omega}^{-1} \sum_{i=1}^{n} Z_{i}\left(\hat{e}_{i} Z_{i}^{\prime} \widehat{\Omega}^{-1} Z^{\prime} \hat{e}\right) X_{i}^{\prime}, \\
\hat{m}_{i} & =\frac{1}{n} X^{\prime} Z \widehat{\Omega}^{-1} Z_{i} \hat{e}_{i}+\frac{1}{n} X_{i} Z_{i}^{\prime} \widehat{\Omega}^{-1} Z^{\prime} \hat{e}-\frac{1}{n^{2}} X^{\prime} Z \widehat{\Omega}^{-1} Z_{i} Z_{i}^{\prime} \hat{e}_{i}^{2} \widehat{\Omega}^{-1} Z^{\prime} \hat{e} .
\end{aligned}
$$

In comparison, the Windmeijer corrected and the conventional variance estimators are

$$
\begin{aligned}
\widehat{V}_{w}(\hat{\theta}) & =\widehat{H}^{-1}\left(\frac{1}{n^{2}} X^{\prime} Z \widehat{\Omega}^{-1} Z^{\prime} X\right) \widehat{H}^{-1^{\prime}}, \\
\widetilde{V}(\hat{\theta}) & =\left(\frac{1}{n^{2}} X^{\prime} Z \widehat{\Omega}^{-1} Z^{\prime} X\right)^{-1} .
\end{aligned}
$$

\subsection{A Panel Data Model}

Consider a panel data model with a scalar regressor

$$
y_{i t}=x_{i t} \beta+\eta_{i}+v_{i t}
$$

for $i=1, \ldots, N$ and $t=1, \ldots, T$ where $\eta_{i}$ is the unobserved individual effects, the unknown parameter of interest is $\beta$, and the single regressor $x_{i t}$ is predetermined with respect to $v_{i t}$ (possibly including lags of the dependent variable), i.e., $E\left(x_{i t} v_{i s}\right)=0$ for all $s \geq t$. After first-differencing,

$$
\Delta y_{i t}=\Delta x_{i t} \beta+\Delta v_{i t}, \quad t=2, \ldots, T,
$$


the standard approach to estimate $\beta$ is the first differenced GMM (Arellano and Bond (1991) estimator) with the moment conditions $E\left(Z_{i}^{\prime} \Delta v_{i}\right)=0$ where $Z_{i}$ is the $(T-1) \times T(T-1) / 2$ instrument matrix

$$
Z_{i}=\operatorname{diag}\left(z_{i 2}^{\prime}, \cdots, z_{i T}^{\prime}\right)
$$

with all possible lagged instruments $z_{i t}=\left(x_{i 1}, \cdots, x_{i t-1}\right)^{\prime}$ for $2 \leq t \leq T$ and $\Delta v_{i}=\left(\Delta v_{i 2}, \cdots, \Delta v_{i T}\right)^{\prime}$. The total number of observations is $n=N(T-1)$.

Our doubly corrected variance estimator can be used for the model (74) with additional strictly exogenous, predetermined, or endogenous variables as well as the system GMM estimator (Arellano and Bover (1995) and Blundell and Bond (1998)) by stacking and modifying additional moment conditions into the instrument sets $Z_{i}$. If the panel is unbalanced the instrument matrix can be constructed as described in Arellano and Bond (1991).

Using the initial weight matrix $\widehat{W}=n^{-1} \sum_{i=1}^{N} Z_{i}^{\prime} H Z_{i}$, where $H$ is a matrix with 2's on the main diagonal, -1 's on the first off-diagonals and zero elsewhere, the one-step GMM estimator is given by

$$
\hat{\beta}_{1}=\left(\Delta X^{\prime} Z \widehat{W}^{-1} Z^{\prime} \Delta X\right)^{-1} \Delta X^{\prime} Z \widehat{W}^{-1} Z^{\prime} \Delta Y
$$

where $Z=\left(Z_{1}^{\prime}, \ldots, Z_{N}^{\prime}\right)^{\prime}$ is the instrument matrix, $\Delta Y=\left(\Delta y_{1}^{\prime}, \ldots, \Delta y_{N}^{\prime}\right)^{\prime}, \Delta X=\left(\Delta x_{1}^{\prime}, \ldots, \Delta x_{N}^{\prime}\right)^{\prime}$, $\Delta y_{i}=\left(\Delta y_{i 2}, \ldots, \Delta y_{i T}\right)^{\prime}$, and $\Delta x_{i}=\left(\Delta x_{i 2}, \ldots, \Delta x_{i T}\right)^{\prime}$. Note that scaling the weight matrix does not affect the estimator. The doubly corrected variance estimator of $\hat{\beta}_{1}$ is given by

$$
\begin{aligned}
\widehat{V}_{d c}\left(\hat{\beta}_{1}\right) & =n^{2}\left(\Delta X^{\prime} Z \widehat{W}^{-1} Z^{\prime} \Delta X\right)^{-1}\left(\frac{1}{n} \sum_{i=1}^{N} \hat{m}_{1 i} \hat{m}_{1 i}^{\prime}\right)\left(\Delta X^{\prime} Z \widehat{W}^{-1} Z^{\prime} \Delta X\right)^{-1}, \\
\hat{m}_{1 i} & =\Delta X^{\prime} Z \widehat{W}^{-1} Z_{i}^{\prime} \Delta \hat{v}_{1 i}+\Delta x_{i}^{\prime} Z_{i} \widehat{W}^{-1} Z^{\prime} \Delta \hat{v}_{1}-\frac{1}{n} \Delta X^{\prime} Z \widehat{W}^{-1} Z_{i}^{\prime} H Z_{i} \widehat{W}^{-1} Z^{\prime} \Delta \hat{v}_{1},
\end{aligned}
$$

where $\Delta \hat{v}_{1 i}=\Delta y_{i}-\Delta x_{i} \hat{\beta}_{1}$ and $\Delta \hat{v}_{1}=\left(\Delta \hat{v}_{11}^{\prime}, \ldots, \Delta \hat{v}_{1 N}^{\prime}\right)^{\prime}$. The doubly corrected standard error is

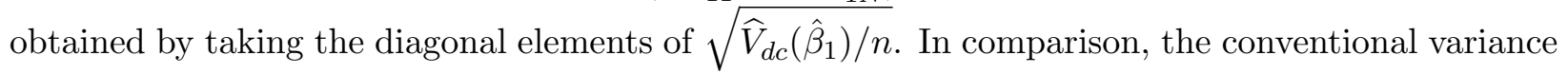
estimator is given by

$$
\widetilde{V}\left(\hat{\beta}_{1}\right)=n^{2}\left(\Delta X^{\prime} Z \widehat{W}^{-1} Z^{\prime} \Delta X\right)^{-1} \Delta X^{\prime} Z \widehat{W}^{-1} \widehat{\Omega}_{1} \widehat{W}^{-1} Z^{\prime} \Delta X\left(\Delta X^{\prime} Z \widehat{W}^{-1} Z^{\prime} \Delta X\right)^{-1}
$$

where

$$
\widehat{\Omega}_{1}=\frac{1}{n} \sum_{i=1}^{N} Z_{i}^{\prime} \Delta \hat{v}_{1 i} \Delta \hat{v}_{1 i}^{\prime} Z_{i} .
$$

Next, consider the two-step efficient GMM estimator

$$
\hat{\beta}_{2}=\left(\Delta X^{\prime} Z \widehat{\Omega}_{1}^{-1} Z^{\prime} \Delta X\right)^{-1} \Delta X^{\prime} Z \widehat{\Omega}_{1}^{-1} Z^{\prime} \Delta Y .
$$

Let $\Delta \hat{v}_{2 i}=\Delta y_{i}-\Delta x_{i} \hat{\beta}_{2}$ and $\Delta \hat{v}_{2}=\left(\Delta \hat{v}_{21}^{\prime}, \ldots, \Delta \hat{v}_{2 N}^{\prime}\right)^{\prime}$. The doubly corrected variance estimator of 
$\hat{\beta}_{2}$ is given by

$$
\widehat{V}_{d c}\left(\hat{\beta}_{2}\right)=\widehat{V}\left(\hat{\beta}_{2}\right)+\widehat{D}_{n} \widehat{C}\left(\hat{\beta}_{1}, \hat{\beta}_{2}\right)+\widehat{C}\left(\hat{\beta}_{1}, \hat{\beta}_{2}\right)^{\prime} \widehat{D}_{n}^{\prime}+\widehat{D}_{n} \widehat{V}_{d c}\left(\hat{\beta}_{1}\right) \widehat{D}_{n}^{\prime},
$$

where

$$
\begin{aligned}
\widehat{V}\left(\hat{\beta}_{2}\right) & =n^{2}\left(\Delta X^{\prime} Z \widehat{\Omega}_{1}^{-1} Z^{\prime} \Delta X\right)^{-1}\left(\frac{1}{n} \sum_{i=1}^{N} \hat{m}_{2 i} \hat{m}_{2 i}^{\prime}\right)\left(\Delta X^{\prime} Z \widehat{\Omega}_{1}^{-1} Z^{\prime} \Delta X\right)^{-1}, \\
\widehat{C}\left(\hat{\beta}_{1}, \hat{\beta}_{2}\right) & =n^{2}\left(\Delta X^{\prime} Z \widehat{W}^{-1} Z^{\prime} \Delta X\right)^{-1}\left(\frac{1}{n} \sum_{i=1}^{N} \hat{m}_{1 i} \hat{m}_{2 i}^{\prime}\right)\left(\Delta X^{\prime} Z \widehat{\Omega}_{1}^{-1} Z^{\prime} \Delta X\right)^{-1}, \\
\hat{m}_{2 i} & =\Delta X^{\prime} Z \widehat{\Omega}_{1}^{-1} Z_{i}^{\prime} \Delta \hat{v}_{2 i}+\Delta x_{i}^{\prime} Z_{i} \widehat{\Omega}_{1}^{-1} Z^{\prime} \Delta \hat{v}_{2}-\frac{1}{n} \Delta X^{\prime} Z \widehat{\Omega}_{1}^{-1} Z_{i}^{\prime} \Delta \hat{v}_{1 i} \Delta \hat{v}_{1 i}^{\prime} Z_{i} \widehat{\Omega}_{1}^{-1} Z^{\prime} \Delta \hat{v}_{2}, \\
\widehat{D}_{n} & =\left(\Delta X^{\prime} Z \widehat{\Omega}_{1}^{-1} Z^{\prime} \Delta X\right)^{-1} \Delta X^{\prime} Z \widehat{\Omega}_{1}^{-1} \\
& \times \frac{1}{n} \sum_{i=1}^{N}\left(Z_{i}^{\prime} \Delta x_{i}\left(\Delta \hat{v}_{2}^{\prime} Z \widehat{\Omega}_{1}^{-1} Z_{i}^{\prime} \Delta \hat{v}_{1 i}\right)+\left(Z_{i}^{\prime} \Delta \hat{v}_{1 i}\right)\left(\Delta \hat{v}_{2}^{\prime} Z \widehat{\Omega}_{1}^{-1} Z_{i}^{\prime} \Delta x_{i}\right)\right) .
\end{aligned}
$$

The doubly corrected standard error is obtained by taking the diagonal elements of $\sqrt{\widehat{V}_{d c}\left(\hat{\beta}_{2}\right) / n}$. Note that the Windmeijer corrected variance estimator is

$$
\widehat{V}_{w}\left(\hat{\beta}_{2}\right)=\widetilde{V}\left(\hat{\beta}_{2}\right)+\widehat{D}_{n} \widetilde{V}\left(\hat{\beta}_{2}\right)+\widetilde{V}\left(\hat{\beta}_{2}\right) \widehat{D}_{n}^{\prime}+\widehat{D}_{n} \widetilde{V}\left(\hat{\beta}_{1}\right) \widehat{D}_{n}^{\prime},
$$

where

$$
\widetilde{V}\left(\hat{\beta}_{2}\right)=n^{2}\left(\Delta X^{\prime} Z \widehat{\Omega}_{1}^{-1} Z^{\prime} \Delta X\right)^{-1} .
$$

Finally, the iterated GMM estimator is given as follows. Let $\hat{\beta}_{0}$ be any initial value. The $s$-step GMM estimator for $s \geq 1$ is given by

$$
\hat{\beta}_{s}=\left(\Delta X^{\prime} Z \widehat{\Omega}_{s-1}^{-1} Z^{\prime} \Delta X\right)^{-1} \Delta X^{\prime} Z \widehat{\Omega}_{s-1}^{-1} Z^{\prime} \Delta Y
$$

where

$$
\widehat{\Omega}_{s-1}=\frac{1}{n} \sum_{i=1}^{N} Z_{i}^{\prime}\left(\Delta y_{i}-\Delta x_{i} \hat{\beta}_{s-1}\right)\left(\Delta y_{i}-\Delta x_{i} \hat{\beta}_{s-1}\right)^{\prime} Z_{i} .
$$

We iterate the $s$-step GMM estimator until convergence given a preset tolerance, e.g. $\left\|\hat{\beta}_{s}-\hat{\beta}_{s-1}\right\|<$ $10^{-5}$ to obtain the iterated GMM estimator $\hat{\beta}$. The residuals are $\Delta \hat{v}_{i}=\Delta y_{i}-\Delta x_{i} \hat{\beta}$. Also let $\Delta \hat{v}=\left(\Delta \hat{v}_{1}^{\prime}, \ldots, \Delta \hat{v}_{N}^{\prime}\right)^{\prime}$ be the $n \times 1$ residual vector. 
The doubly corrected variance estimator for the iterated GMM is given by

$$
\begin{aligned}
\widehat{V}_{d c}(\hat{\beta})= & \widehat{H}^{-1}\left(\frac{1}{n} \sum_{i=1}^{N} \hat{m}_{i} \hat{m}_{i}^{\prime}\right) \widehat{H}^{-1^{\prime}} \\
\widehat{H}= & \frac{1}{n^{2}} \Delta X^{\prime} Z \widehat{\Omega}^{-1} Z^{\prime} \Delta X \\
& -\frac{1}{n^{3}} \Delta X^{\prime} Z \widehat{\Omega}^{-1}\left(\sum_{i=1}^{N}\left(Z_{i}^{\prime} \Delta \hat{v}_{i}\right)\left(\Delta \hat{v}^{\prime} Z \widehat{\Omega}^{-1} Z_{i}^{\prime} \Delta x_{i}\right)+Z_{i}^{\prime} \Delta x_{i}\left(\Delta \hat{v}^{\prime} Z \widehat{\Omega}^{-1} Z_{i}^{\prime} \Delta \hat{v}_{i}\right)\right), \\
\hat{m}_{i}= & \frac{1}{n} \Delta X^{\prime} Z \widehat{\Omega}^{-1} Z_{i}^{\prime} \Delta \hat{v}_{i}+\frac{1}{n} \Delta X_{i}^{\prime} Z_{i} \widehat{\Omega}^{-1} Z^{\prime} \Delta \hat{v}-\frac{1}{n^{2}} \Delta X^{\prime} Z \widehat{\Omega}^{-1} Z_{i}^{\prime} \Delta \hat{v}_{i} \Delta \hat{v}_{i}^{\prime} Z_{i} \widehat{\Omega}^{-1} Z^{\prime} \Delta \hat{v}
\end{aligned}
$$

and the doubly corrected standard error is obtained by taking the diagonal elements of $\sqrt{\widehat{V}_{d c}(\hat{\beta}) / n}$.

In comparison, the Windmeijer corrected and the conventional variance estimators are

$$
\begin{aligned}
\widehat{V}_{w}(\hat{\beta}) & =\widehat{H}^{-1}\left(\frac{1}{n^{2}} \Delta X^{\prime} Z \widehat{\Omega}^{-1} Z^{\prime} \Delta X\right) \widehat{H}^{-1^{\prime}}, \\
\widetilde{V}(\hat{\beta}) & =\left(\frac{1}{n^{2}} \Delta X^{\prime} Z \widehat{\Omega}^{-1} Z^{\prime} \Delta X\right)^{-1}
\end{aligned}
$$

\section{Simulation}

We investigate the finite sample performance of the doubly corrected standard errors proposed in this paper and provide a thorough comparison with the conventional and the Windmeijer corrected ones under correct specification and misspecification. We consider three different setups: (i) a cross-sectional linear IV model with potentially invalid instruments; (ii) a linear dynamic panel model with a random coefficient; (iii) a linear dynamic panel model with possibly misspecified lag specifications. The number of Monte Carlo simulation is 100,000.

In an unreported simulation, we also investigate the performance of the estimators with the centered weight matrix (58). Since the results are similar and there is no obvious pattern of better performance of the point and variance estimators based on the centered weight matrix compared with those based on the uncentered one (reported) they are not reported.

\subsection{Cross-sectional IV}

We use the following simulation design which is a simple linear instrumental variable regression with a single endogenous regressor. The model to be estimated is

$$
\begin{aligned}
y_{i} & =x_{i} \beta_{0}+e_{i} \\
E\left(z_{i} e_{i}\right) & =0
\end{aligned}
$$

where $x_{i}$ and $\beta_{0}$ are scalar and $z_{i}=\left(z_{1 i}, z_{2 i}, z_{3 i}, z_{4 i}\right)^{\prime}$ is a vector of instrumental variables. We estimate $\beta_{0}$ by 2SLS (one-step), two-step, and iterated GMM, and calculate the conventional, the 
Windmeijer corrected, and the doubly corrected standard errors. Our data-generating process (DGP) is

$$
\begin{aligned}
y_{i} & =x_{i} \beta_{0}+\frac{\alpha_{0}}{\sqrt{n}}\left(z_{1 i}-z_{2 i}+z_{3 i}-z_{4 i}\right)+e_{i}, \\
x_{i} & =\pi_{0}\left(z_{1 i}+z_{2 i}+z_{3 i}+z_{4 i}\right)+u_{i}, \\
e_{i} & =0.5 u_{i}+\sqrt{1-0.5^{2}} v_{i}, \\
z_{i} & \sim N\left(0, I_{4}\right),\left(\begin{array}{c}
u_{i} \\
v_{i}
\end{array}\right) \sim N\left(\left(\begin{array}{l}
0 \\
0
\end{array}\right),\left[\begin{array}{cc}
1 & 0 \\
0 & z_{1 i}^{2}
\end{array}\right]\right) .
\end{aligned}
$$

We set $\beta_{0}=1$, vary $\alpha_{0}$ from 0 to 1 in steps of 0.2 , and set the first-stage coefficient $\pi_{0}$ so that the first-stage $R^{2}=0.2$. We set the number of observations as $n=50,100,500$.

The parameter $\alpha_{0}$ is the extent that the exclusion condition is locally violated. At $\alpha_{0}=0$, the model is correctly specified. For $\alpha_{0} \neq 0$, we find $E\left(z_{i} e_{i}\right)=\left(\alpha_{0},-\alpha_{0}, \alpha_{0},-\alpha_{0}\right)^{\prime} / \sqrt{n} \neq 0$, so the moment condition (80) fails to hold in finite samples, but it holds asymptotically.

Means and standard deviations of one-step (2SLS), two-step, and iterated GMM estimators are computed in Table 1. For all GMM estimators, we report means of the conventional standard

errors (se $\hat{\beta}$ ), the Windmeijer corrected standard errors $\left(\operatorname{se}_{w} \hat{\beta}\right)$, and the doubly corrected standard errors $\left(\operatorname{se}_{d c} \hat{\beta}\right)$.

Table 1 shows that our doubly corrected standard errors remains accurate regardless of misspecification, including the correct specification case $\left(\alpha_{0}=0\right)$; the means of corrected standard errors are very close to the standard deviations for all values of $\alpha_{0}$, especially for the two-step and the iterated GMM. Simulation evidence reassures our theory that the doubly corrected standard errors not only take into account variation in the estimation of the weight matrix, but also extra variation due to the non-zero sample moments in over-identified model even under correct specification. Furthermore, our doubly corrected standard errors are the only valid one under misspecification.

The conventional standard error for the one-step GMM (2SLS) estimator is downward biased under correct specification $\left(\alpha_{0}=0\right)$, and this bias increases with $\alpha_{0}$. As is well known, the conventional standard error for the two-step is severely downward biased when $\alpha_{0}=0$, and this bias also increases with $\alpha_{0}$. The Windmeijer corrected standard error works well under correct specification, but does not fully account for additional variations due to non-zero $\alpha_{0}$. The result is similar for the iterated GMM.

\subsection{Linear Dynamic Panel Model}

\subsubsection{Random Coefficient}

We next explore the finite sample performance of the doubly corrected standard error in the presence of heterogeneous effects (random coefficient) in dynamic panel model. We consider the 
AR(1) dynamic panel model of Blundell and Bond (1998). For $i=1, \ldots, N$ and $t=1, \ldots, T$,

$$
y_{i t}=\rho_{0} y_{i, t-1}+\eta_{i}+\nu_{i t},
$$

where $\eta_{i}$ is an unobserved individual-specific effect and $\nu_{i t}$ is an error term. The parameter of interest $\rho_{0}$ is estimated by the difference GMM based on a set of moment conditions:

$$
E\left[y_{i, t-s}\left(\Delta y_{i t}-\rho_{0} \Delta y_{i, t-1}\right)\right]=0, \quad t=3, \ldots T, \text { and } s \geq 2,
$$

The moment conditions are derived from taking differences of (82), and uses the lagged values of $y_{i t}$ as instruments. The number of moment conditions is $(T-1)(T-2) / 2$.

The moment conditions are correctly specified if there is a unique parameter that satisfies (83). A sufficient condition for this to hold is that the model (82) coincides with the true DGP, but this is unlikely to be true. A reasonable deviation from the assumed model (82) is heterogeneity in $\rho_{0}$ across $i$. We assume the following DGP. For $i=1, \ldots, N$ and $t=1, \ldots T$,

$$
\begin{aligned}
& y_{i t}=\rho_{i} y_{i, t-1}+\eta_{i}+\nu_{i t}, \\
& \eta_{i} \sim N(0,1) ; \rho_{i} \sim \Phi\left(\alpha_{0} \eta_{i}\right) ; \nu_{i t} \sim N\left(0,0.5^{2}\right), \\
& y_{i 1}=\frac{\eta_{i}}{1-\rho_{i}}+u_{i 1} ; u_{i 1} \sim N\left(0, \frac{1}{1-\rho_{i}^{2}}\right),
\end{aligned}
$$

where $\Phi(z)$ is the standard normal cdf. At $\alpha_{0}=0$, the model is correctly specified and $\rho_{i}=\rho_{0}=0.5$. For $\alpha_{0} \neq 0$, the effective moment condition model can be written as

$$
\begin{aligned}
E\left[y_{i, t-s}\left(\Delta y_{i t}-\rho \Delta y_{i, t-1}\right)\right] & =E\left[y_{i, t-s}\left(\Delta \nu_{i t}+\left(\rho_{i}-\rho\right) \Delta y_{i, t-1}\right)\right] \\
& =E\left[\rho_{i} y_{i, t-s} \Delta y_{i, t-1}\right]-\rho\left(\gamma_{s-1}-\gamma_{s-2}\right)
\end{aligned}
$$

where $\gamma_{j}$ is the $j$ th autocovariance. The last equation becomes zero at $\rho=E\left[\rho_{i}\right]$ if $\rho_{i}$ is independent of the $\left\{y_{i t}\right\}$ process. If this is the case, then the moment condition model is correctly specified and the estimand is $E\left[\rho_{i}\right]$. Otherwise in general, the moment condition model fails to hold at a single unique parameter value because each of the moment condition imposes a restriction

$$
\rho=\frac{E\left[\rho_{i} y_{i, t-s} \Delta y_{i, t-1}\right]}{\gamma_{s-1}-\gamma_{s-2}}
$$

but there is no reason that this should hold at a unique $\rho$ for $s=2,3, \ldots, t-1$. In the DGP, $\eta_{i}$ and $\rho_{i}$ are dependent through $\alpha_{0}$ and a larger $\alpha_{0}$ leads to larger heterogeneity. We vary $\alpha_{0}$ from 0 to 0.3 in steps of 0.05. The pseudo-true value would depend on the instrument set and the value of $\alpha_{0}$ under global misspecification. However, by varying $\alpha_{0}$ by a small amount we try to capture local behavior of the standard errors when the pseudo-true value is close to the true value. The sample sizes are $N=100,500$ and $T=4,6$.

We report the simulation results in Tables 2 and 3 , which are qualitatively similar to the IV 
setup. Tables 2 and 3 show that the doubly corrected standard errors approximate the standard deviation of the GMM estimators well regardless of misspecification. For the two-step and iterated GMM estimators, the doubly corrected standard errors are as accurate as the Windmeijer correction for small values of $\alpha_{0}$ (including correct specification $\alpha_{0}=0$ ) but dominate the other in accuracy for larger values of $\alpha_{0}$. The doubly corrected standard error for the one-step GMM is slightly upward biased for small values of $\alpha_{0}$, but this bias decreases with a larger sample size $N=500$.

\subsubsection{Misspecified Lag Length}

We use the baseline linear panel model of Windmeijer (2005) allowing for possible lag length misspecification. The model is

$$
y_{i t}=\beta_{0} x_{i t}+\eta_{i}+v_{i t},
$$

for $i=1, \ldots, N$ and $t=1, \ldots, T$. The unknown parameter of interest is $\beta_{0}$, and the regressor $x_{i t}$ is predetermined with respect to $v_{i t}$, i.e., $E\left(x_{i t} v_{i t+s}\right)=0$ for $s=0, \ldots, T-t$. We use the first differenced GMM estimator and the number of moment conditions is $T(T-1) / 2$ as in Section 7.2.

The DGP is

$$
\begin{aligned}
y_{i t} & =\beta_{0} x_{i t}+\alpha_{0} x_{i t-1}+\eta_{i}+v_{i t}, \\
x_{i t} & =0.5 x_{i t-1}+\eta_{i}+0.5 v_{i t-1}+\epsilon_{i t}, \\
\eta & \sim N(0,1) \text { and } \epsilon_{i t} \sim N(0,1), \\
v_{i t} & =\delta_{i} \tau_{t} \omega_{i t} \text { and } \omega_{i t} \sim \chi_{1}^{2}-1 .
\end{aligned}
$$

We generate initial 50 time periods with $\tau_{t}=0.5$ for $t=-49, \ldots, 0$ and $x_{i} \sim N\left(\eta_{i} / 0.5,1 / 0.75\right)$ same as Windmeijer (2005). The parameter $\alpha_{0}$ in (85) governs the degree of misspecification. When $\alpha_{0}=0$, the model (84) is correctly specified which reduces to that of Windmeijer (2005).

Tables 4 and 5 report estimation results for $\beta_{0}=1, N=100,500$ and $T=4,6$. The degree of misspecification $\alpha_{0}$ is varied across $\{0,0.025,0.05,0.1\}$. The first column $\left(\alpha_{0}=0\right)$ in Table 4 replicates Monte Carlo studies in Windmeijer (2005, Table 1).

The implication of the results in Tables 4 and 5 are largely unchanged as in two previous simulation experiments; doubly corrected standard errors approximate the standard deviations well regardless of model misspecification. In this simulation experiments, the Windmeijer correction works best under correct specification, but becomes downward biased as $\alpha_{0}$ increases. Note that deviation from the correct specification makes the bias of the conventional standard error and the Windmeijer corrected standard error larger, and this bias does not disappear with a larger sample size of $N=500$. 


\section{Appendix: Proofs}

In the proof of Theorems 1 and 2, we use following lemma on geometric expansion of a matrix. Lemma 1 builds on Corollary 1 of Magdalinos (1992).

Lemma 1. Let $X_{n}$ and $Y_{n}$ be square random matrices. If $X_{n}^{-1}$ and $\left(X_{n}+Y_{n} / \sqrt{n}\right)^{-1}$ exist and $X_{n}^{-1}$ and $Y_{n}$ are of order $O_{p}(1)$, then following holds for any nonnegative integer $q$,

$$
\left(X_{n}+\frac{1}{\sqrt{n}} Y_{n}\right)^{-1}=\sum_{j=0}^{q}\left(-\frac{1}{\sqrt{n}} X_{n}^{-1} Y_{n}\right)^{j} X_{n}^{-1}+O_{p}\left(n^{-(q+1) / 2}\right) .
$$

Proof of Lemma 1: Let $S_{n}=X_{n}^{-1} Y_{n}$ and consider the following identity,

$$
\sum_{j=0}^{q}\left(-\frac{1}{\sqrt{n}}\right)^{j} S_{n}^{j}\left(I+\frac{1}{\sqrt{n}} S_{n}\right)=I-\left(-\frac{1}{\sqrt{n}}\right)^{q+1} S_{n}^{q+1}
$$

Using $I+n^{-1 / 2} S_{n}=X_{n}^{-1}\left(X_{n}+n^{-1 / 2} Y_{n}\right), S_{n}=X_{n}^{-1} Y_{n}$ and rearranging terms, we have

$$
\begin{aligned}
\left(X_{n}+\frac{1}{\sqrt{n}} Y_{n}\right)^{-1} & =\sum_{j=0}^{q}\left(-\frac{1}{\sqrt{n}}\right)^{j} S_{n}^{j} X_{n}^{-1}+\left(-\frac{1}{\sqrt{n}}\right)^{q+1} S_{n}^{q+1}\left(X_{n}+\frac{1}{\sqrt{n}} Y_{n}\right)^{-1} \\
& =\sum_{j=0}^{q}\left(-\frac{1}{\sqrt{n}}\right)^{j}\left(X_{n}^{-1} Y_{n}\right)^{j} X_{n}^{-1}+\left(-\frac{1}{\sqrt{n}}\right)^{q+1} O_{p}(1)
\end{aligned}
$$

by the assumptions of the lemma.

Proof of Theorem 1: In what following the statements hold with probability approaching one. First note that

$$
\begin{aligned}
G_{n}^{\prime} W_{n}^{-1} G_{n} & =G^{\prime} W^{-1} G+\left(G_{n}-G\right)^{\prime} W^{-1} G+G^{\prime} W^{-1}\left(G_{n}-G\right)+G^{\prime}\left(W_{n}^{-1}-W^{-1}\right) G+O_{p}\left(n^{-1}\right) \\
& =G^{\prime} W^{-1} G+O_{p}\left(n^{-1 / 2}\right) .
\end{aligned}
$$

By Lemma 1 with $q=1$,

$$
\begin{aligned}
\left(G_{n}^{\prime} W_{n}^{-1} G_{n}\right)^{-1}= & \left(G^{\prime} W^{-1} G+\frac{1}{\sqrt{n}} \sqrt{n}\left(G_{n}^{\prime} W_{n}^{-1} G_{n}-G^{\prime} W^{-1} G\right)\right)^{-1} \\
= & \left(G^{\prime} W^{-1} G\right)^{-1}-\frac{1}{\sqrt{n}}\left(G^{\prime} W^{-1} G\right)^{-1} \sqrt{n}\left(G_{n}^{\prime} W_{n}^{-1} G_{n}-G^{\prime} W^{-1} G\right)\left(G^{\prime} W^{-1} G\right)^{-1} \\
& +O_{p}\left(n^{-1}\right) \\
= & \left(G^{\prime} W^{-1} G\right)^{-1}+\frac{1}{\sqrt{n}} \widetilde{B}_{W}\left(G^{\prime} W^{-1} G\right)^{-1}+O_{p}\left(n^{-1}\right) .
\end{aligned}
$$


In addition,

$$
\begin{aligned}
G_{n}^{\prime} W_{n}^{-1} \sqrt{n} g_{n}\left(\theta_{0}\right)= & G_{n}^{\prime} W_{n}^{-1} \sqrt{n}\left(g_{n}\left(\theta_{0}\right)-g\right)+\sqrt{n}\left(G_{n}-G\right)^{\prime} W_{n}^{-1} g+G^{\prime} \sqrt{n}\left(W_{n}^{-1}-W^{-1}\right) g+G^{\prime} W^{-1} \delta \\
= & G^{\prime} W^{-1} \widetilde{g}+\widetilde{G}^{\prime} W^{-1} g-G^{\prime} W^{-1} \widetilde{W} W^{-1} g+\widetilde{G}^{\prime} W^{-1} \widetilde{g} / \sqrt{n}-G^{\prime} W^{-1} \widetilde{W} W^{-1} \widetilde{g} / \sqrt{n} \\
& +G^{\prime} W^{-1} \delta+O_{p}\left(n^{-1}\right) .
\end{aligned}
$$

Since the FOC of the one-step GMM holds regardless of misspecification of the moment condition, we use the above expansions to obtain

$$
\begin{aligned}
& \sqrt{n}\left(\hat{\theta}_{1}-\theta_{0}\right) \\
= & \left(G_{n} W_{n}^{-1} G_{n}\right)^{-1} G_{n} W_{n}^{-1} \sqrt{n} g_{n}\left(\theta_{0}\right) \\
= & -\left[\left(G^{\prime} W^{-1} G\right)^{-1}+\widetilde{B}_{W}\left(G^{\prime} W^{-1} G\right)^{-1} / \sqrt{n}\right] \times\left[G^{\prime} W^{-1} \widetilde{g}+\widetilde{G}^{\prime} W^{-1} g-G^{\prime} W^{-1} \widetilde{W} W^{-1} g\right. \\
& \left.+\widetilde{G}^{\prime} W^{-1} \widetilde{g} / \sqrt{n}-G^{\prime} W^{-1} \widetilde{W} W^{-1} \widetilde{g} / \sqrt{n}+G^{\prime} W^{-1} \delta\right]+O_{p}\left(n^{-1}\right) \\
= & \eta_{W}+\widetilde{B}_{W} \eta_{W} / \sqrt{n}-\left(G^{\prime} W^{-1} G\right)^{-1} G^{\prime} W^{-1} \widetilde{g}-\left(G^{\prime} W^{-1} G\right)^{-1}\left(\widetilde{G}^{\prime} W^{-1} g-G^{\prime} W^{-1} \widetilde{W} W^{-1} g\right) \\
& -\left(G^{\prime} W^{-1} G\right)^{-1}\left(\widetilde{G} W^{-1} \widetilde{g}-G^{\prime} W^{-1} \widetilde{W} W^{-1} \widetilde{g}\right) / \sqrt{n}-\widetilde{B}_{W}\left(G^{\prime} W^{-1} G\right)^{-1} G^{\prime} W^{-1} \widetilde{g} / \sqrt{n}+O_{p}\left(n^{-1}\right) \\
= & \eta_{W}+\widetilde{\psi}_{W, 0}+\left(\widetilde{\psi}_{W, 1}+\widetilde{q}_{W}+\widetilde{B}_{W}\left(\widetilde{\psi}_{W, 0}+\eta_{W}\right)\right) / \sqrt{n}+O_{p}\left(n^{-1}\right) .
\end{aligned}
$$

Proof of Theorem 2: In what following the statements hold with probability approaching one. We first prove some useful expansions. Note that from Theorem $1, \sqrt{n}\left(\hat{\theta}_{1}-\theta_{0}\right)=O_{p}(1)$. By the first-order Taylor expansion and (13),

$$
\begin{aligned}
\Omega_{n}\left(\hat{\theta}_{1}\right) & =\Omega_{n}\left(\theta_{0}\right)+\frac{1}{\sqrt{n}} \sum_{j} \frac{\partial \Omega(\theta)}{\partial \theta_{[j]}} \sqrt{n}\left(\hat{\theta}_{1[j]}-\theta_{0[j]}\right) \\
& =\Omega_{n}\left(\theta_{0}\right)-\Omega+\Omega+\frac{1}{\sqrt{n}} \sum_{j} \frac{\partial \Omega(\theta)}{\partial \theta_{[j]}} \sqrt{n}\left(\hat{\theta}_{1[j]}-\theta_{0[j]}\right) \\
& =\Omega+O_{p}\left(n^{-1 / 2}\right) .
\end{aligned}
$$

By Lemma 1 with $q=0$,

$$
\begin{aligned}
& {\left[\Omega_{n}\left(\hat{\theta}_{1}\right)\right]^{-1}=\Omega^{-1}+O_{p}\left(n^{-1 / 2}\right),} \\
& {\left[\Omega_{n}\left(\theta_{0}\right)\right]^{-1}=\Omega^{-1}+O_{p}\left(n^{-1 / 2}\right) .}
\end{aligned}
$$


Using (88) and applying Lemma 1 with $q=1$ to (86),

$$
\begin{aligned}
{\left[\Omega_{n}\left(\hat{\theta}_{1}\right)\right]^{-1} } & =\left[\Omega_{n}\left(\theta_{0}\right)\right]^{-1}-\frac{1}{\sqrt{n}}\left[\Omega_{n}\left(\theta_{0}\right)\right]^{-1} \sum_{j} \frac{\partial \Omega(\theta)}{\partial \theta_{[j]}} \sqrt{n}\left(\hat{\theta}_{1[j]}-\theta_{0[j]}\right)\left[\Omega_{n}\left(\theta_{0}\right)\right]^{-1}+O_{p}\left(n^{-1}\right) \\
& =\left[\Omega_{n}\left(\theta_{0}\right)\right]^{-1}-\frac{1}{\sqrt{n}} \Omega^{-1} \sum_{j} \frac{\partial \Omega(\theta)}{\partial \theta_{[j]}} \sqrt{n}\left(\hat{\theta}_{1[j]}-\theta_{0[j]}\right) \Omega^{-1}+O_{p}\left(n^{-1}\right)
\end{aligned}
$$

Using a similar argument with the proof of Theorem 1 and Lemma 1 with $q=1$, we obtain

$$
\begin{aligned}
\left(G_{n}^{\prime}\left[\Omega_{n}\left(\theta_{0}\right)\right]^{-1} G_{n}\right)^{-1}= & \left(G^{\prime} \Omega^{-1} G\right)^{-1}+\frac{1}{\sqrt{n}} B_{\Omega}\left(G^{\prime} \Omega^{-1} G\right)^{-1}+O_{p}\left(n^{-1}\right), \\
G_{n}^{\prime}\left[\Omega_{n}\left(\theta_{0}\right)\right]^{-1} \sqrt{n} g_{n}\left(\theta_{0}\right)= & G^{\prime} \Omega^{-1} \delta+G^{\prime} \Omega^{-1} \widetilde{g}+\widetilde{G}^{\prime} \Omega^{-1} g-G^{\prime} \Omega^{-1} \widetilde{\Omega} \Omega^{-1} g \\
& +\widetilde{G} \Omega^{-1} \widetilde{g} / \sqrt{n}-G^{\prime} \Omega^{-1} \widetilde{\Omega} \Omega^{-1} \widetilde{g} / \sqrt{n}+O_{p}\left(n^{-1}\right) .
\end{aligned}
$$

Note that the assumption of the theorem implies that $\hat{\theta}_{2}-\theta_{0}=O_{p}\left(n^{-1 / 2}\right)$. Using (90), the first-order Taylor expansion of the FOC of $\hat{\theta}_{2}$ around $\theta_{0}$ can be written as

$$
\begin{aligned}
0= & G_{n}^{\prime}\left[\Omega_{n}\left(\hat{\theta}_{1}\right)\right]^{-1} g_{n}\left(\theta_{0}\right)+G_{n}^{\prime}\left[\Omega_{n}\left(\hat{\theta}_{1}\right)\right]^{-1} G_{n}\left(\hat{\theta}_{2}-\theta_{0}\right) \\
= & G_{n}^{\prime}\left[\Omega_{n}\left(\theta_{0}\right)\right]^{-1} g_{n}\left(\theta_{0}\right)+G_{n}^{\prime}\left[\Omega_{n}\left(\theta_{0}\right)\right]^{-1} G_{n}\left(\hat{\theta}_{2}-\theta_{0}\right) \\
& +G_{n}^{\prime}\left(\left[\Omega_{n}\left(\hat{\theta}_{1}\right)\right]^{-1}-\left[\Omega_{n}\left(\hat{\theta}_{0}\right)\right]^{-1}\right)\left(g_{n}\left(\theta_{0}\right)+G_{n}\left(\hat{\theta}_{2}-\theta_{0}\right)\right) \\
= & G_{n}^{\prime}\left[\Omega_{n}\left(\theta_{0}\right)\right]^{-1} g_{n}\left(\theta_{0}\right)+G_{n}^{\prime}\left[\Omega_{n}\left(\theta_{0}\right)\right]^{-1} G_{n}\left(\hat{\theta}_{2}-\theta_{0}\right) \\
& -\frac{1}{\sqrt{n}} G^{\prime} \Omega^{-1} \sum_{j} \frac{\partial \Omega(\theta)}{\partial \theta_{[j]}} \sqrt{n}\left(\hat{\theta}_{1[j]}-\theta_{0[j]}\right) \Omega^{-1}\left(g_{n}\left(\theta_{0}\right)+G\left(\hat{\theta}_{2}-\theta_{0}\right)\right)+O_{p}\left(n^{-3 / 2}\right) .
\end{aligned}
$$

By arranging terms, multiplying $\sqrt{n}$, using (91)-(92),

$$
\begin{aligned}
\sqrt{n}\left(\hat{\theta}_{2}-\theta_{0}\right)= & -\left(G_{n}^{\prime}\left[\Omega_{n}\left(\theta_{0}\right)\right]^{-1} G_{n}\right)^{-1} G_{n}^{\prime}\left[\Omega_{n}\left(\theta_{0}\right)\right]^{-1} \sqrt{n} g_{n}\left(\theta_{0}\right) \\
& +\left(G_{n}^{\prime}\left[\Omega_{n}\left(\theta_{0}\right)\right]^{-1} G_{n}\right)^{-1} \\
& \times \frac{1}{\sqrt{n}} G^{\prime} \Omega^{-1} \sum_{j} \frac{\partial \Omega(\theta)}{\partial \theta_{[j]}} \sqrt{n}\left(\hat{\theta}_{1[j]}-\theta_{0[j]}\right) \Omega^{-1}\left(\sqrt{n} g_{n}\left(\theta_{0}\right)+G \sqrt{n}\left(\hat{\theta}_{2}-\theta_{0}\right)\right)+O_{p}\left(n^{-1}\right) .
\end{aligned}
$$

Note that the second term in the RHS is $O_{p}\left(n^{-1 / 2}\right)$. First,

$$
\begin{aligned}
& -\left(G_{n}^{\prime}\left[\Omega_{n}\left(\theta_{0}\right)\right]^{-1} G_{n}\right)^{-1} G_{n}^{\prime}\left[\Omega_{n}\left(\theta_{0}\right)\right]^{-1} \sqrt{n} g_{n}\left(\theta_{0}\right) \\
= & -\left(\left(G^{\prime} \Omega^{-1} G\right)^{-1}+\frac{1}{\sqrt{n}} \widetilde{B}_{\Omega}\left(G^{\prime} \Omega^{-1} G\right)^{-1}\right) \\
& \times\left(G^{\prime} \Omega^{-1} \delta+G^{\prime} \Omega^{-1} \widetilde{g}+\widetilde{G}^{\prime} \Omega^{-1} g-G^{\prime} \Omega^{-1} \widetilde{\Omega} \Omega^{-1} g+\widetilde{G} \Omega^{-1} \widetilde{g} / \sqrt{n}-G^{\prime} \Omega^{-1} \widetilde{\Omega} \Omega^{-1} \widetilde{g} / \sqrt{n}\right) \\
& +O_{p}\left(n^{-1}\right) \\
= & \eta_{\Omega}+\frac{1}{\sqrt{n}} \widetilde{B}_{\Omega} \eta_{\Omega}+\widetilde{\psi}_{\Omega, 0}+\frac{1}{\sqrt{n}}\left(\widetilde{\psi}_{\Omega, 1}+\widetilde{q}_{\Omega}+\widetilde{B}_{\Omega} \widetilde{\psi}_{\Omega, 0}\right)+O_{p}\left(n^{-1}\right) .
\end{aligned}
$$


Thus, we have $\sqrt{n}\left(\hat{\theta}_{2}-\theta_{0}\right)=\eta_{\Omega}+\widetilde{\psi}_{\Omega, 0}+O_{p}\left(n^{-1 / 2}\right)$. Next, using $\sqrt{n}\left(\hat{\theta}_{1}-\theta_{0}\right)=\eta_{W}+\widetilde{\psi}_{W, 0}+O_{p}\left(n^{-1 / 2}\right)$,

$$
\begin{aligned}
& \left(G_{n}^{\prime}\left[\Omega_{n}\left(\theta_{0}\right)\right]^{-1} G_{n}\right)^{-1} \frac{1}{\sqrt{n}} G^{\prime} \Omega^{-1} \sum_{j} \frac{\partial \Omega(\theta)}{\partial \theta_{[j]}} \sqrt{n}\left(\hat{\theta}_{1[j]}-\theta_{0[j]}\right) \Omega^{-1}\left(\sqrt{n} g_{n}\left(\theta_{0}\right)+G \sqrt{n}\left(\hat{\theta}_{2}-\theta_{0}\right)\right) \\
= & \left(G^{\prime} \Omega^{-1} G\right)^{-1} \frac{1}{\sqrt{n}} G^{\prime} \Omega^{-1} \sum_{j} \frac{\partial \Omega(\theta)}{\partial \theta_{[j]}}\left(\eta_{W[j]}+\widetilde{\psi}_{W, 0[j]}\right) \Omega^{-1}\left(\widetilde{g}+\delta+G\left(\eta_{\Omega}+\widetilde{\psi}_{\Omega, 0}\right)\right)+O_{p}\left(n^{-1}\right) \\
= & \left(G^{\prime} \Omega^{-1} G\right)^{-1} \frac{1}{\sqrt{n}} G^{\prime} \Omega^{-1} \sum_{j} \frac{\partial \Omega(\theta)}{\partial \theta_{[j]}} \eta_{W[j]} \Omega^{-1}\left(\widetilde{g}+\delta+G\left(\eta_{\Omega}+\widetilde{\psi}_{\Omega, 0}\right)\right) \\
& +\left(G^{\prime} \Omega^{-1} G\right)^{-1} \frac{1}{\sqrt{n}} G^{\prime} \Omega^{-1} \sum_{j} \frac{\partial \Omega(\theta)}{\partial \theta_{[j]}} \widetilde{\psi}_{W, 0[j]} \Omega^{-1}\left(\widetilde{g}+\delta+G\left(\eta_{\Omega}+\widetilde{\psi}_{\Omega, 0}\right)\right)+O_{p}\left(n^{-1}\right) \\
= & \frac{1}{\sqrt{n}}\left\{\left(D+H_{\eta_{\Omega}}\right) \eta_{W}+\left(\widetilde{C}+H_{\widetilde{\psi}_{\Omega, 0}}\right)\left(\eta_{W}+\widetilde{\psi}_{W, 0}\right)+\left(D+H_{\eta_{\Omega}}\right) \widetilde{\psi}_{W, 0}\right\}+O_{p}\left(n^{-1}\right) .
\end{aligned}
$$

Combining this with (103),

$$
\begin{aligned}
& \sqrt{n}\left(\hat{\theta}_{2}-\theta_{0}\right) \\
= & \eta_{\Omega}+\frac{1}{\sqrt{n}}\left(\left(D+H_{\eta_{\Omega}}\right) \eta_{W}\right)+\widetilde{\psi}_{\Omega, 0} \\
& +\frac{1}{\sqrt{n}}\left(\widetilde{\psi}_{\Omega, 1}+\left(D+\widetilde{C}+H_{\eta_{\Omega}}+H_{\widetilde{\psi}_{\Omega, 0}}\right) \widetilde{\psi}_{W, 0}+\widetilde{q}_{\Omega}+\widetilde{B}_{\Omega}\left(\eta_{\Omega}+\widetilde{\psi}_{\Omega, 0}\right)+\left(\widetilde{C}+H_{\widetilde{\psi}_{\Omega, 0}}\right) \eta_{W}\right) \\
& +O_{p}\left(n^{-1}\right) .
\end{aligned}
$$

Notice that $D \widetilde{\psi}_{W, 1} / n$ in the $O_{p}\left(n^{-1}\right)$ remainder term can be obtained if we plug $\sqrt{n}\left(\hat{\theta}_{1}-\theta_{0}\right)=$ $\eta_{W}+\widetilde{\psi}_{W, 0}+\widetilde{\psi}_{W, 1} / \sqrt{n}+O_{p}\left(n^{-1 / 2}\right)$ into (104). This proves the theorem.

Proof of Theorem 3: We first derive the asymptotic variance matrices of the GMM estimators under misspecification and then show that the doubly corrected variance estimators are consistent. Expanding the FOC of the one-step GMM around the pseudo-true value $\theta_{1}$ we can write

$$
\begin{aligned}
\sqrt{n}\left(\hat{\theta}_{1}-\theta_{1}\right)= & -\left(G^{\prime} W^{-1} G\right)^{-1}\left(G W^{-1} \sqrt{n}\left(g_{n}\left(\theta_{1}\right)-g_{1}\right)+\sqrt{n}\left(G_{n}-G\right)^{\prime} W^{-1} g_{1}\right. \\
& \left.-G^{\prime} W^{-1} \sqrt{n}\left(W_{n}-W\right) W^{-1} g_{1}\right)+O_{p}\left(n^{-1 / 2}\right) \\
= & -\left(G^{\prime} W^{-1} G\right)^{-1}\left(G W^{-1} \sqrt{n} g_{n}\left(\theta_{1}\right)+\sqrt{n} G_{n}^{\prime} W^{-1} g_{1}-G^{\prime} W^{-1} \sqrt{n} W_{n} W^{-1} g_{1}\right) \\
& +O_{p}\left(n^{-1 / 2}\right) .
\end{aligned}
$$

The second equality holds by the population FOC $G^{\prime} W^{-1} g_{1}=0$. Similarly we expand the FOC of 
the two-step GMM as

$$
\begin{aligned}
\sqrt{n}\left(\hat{\theta}_{2}-\theta_{2}\right) & =-\left(G_{n}^{\prime}\left[\Omega_{n}\left(\hat{\theta}_{1}\right)\right]^{-1} G_{n}\right)^{-1} G_{n}\left[\Omega_{n}\left(\hat{\theta}_{1}\right)\right]^{-1} \sqrt{n} g_{n}\left(\theta_{2}\right) \\
& =-\left(G_{n}^{\prime}\left[\Omega_{n}\left(\theta_{1}\right)\right]^{-1} G_{n}\right)^{-1} G_{n}\left[\Omega_{n}\left(\theta_{1}\right)\right]^{-1} \sqrt{n} g_{n}\left(\theta_{2}\right)+D_{n}^{*} \sqrt{n}\left(\hat{\theta}_{1}-\theta_{1}\right)+O_{p}\left(n^{-1}\right)
\end{aligned}
$$

where

$$
\begin{aligned}
& D_{n}^{*}=F_{1 n}^{*}+F_{2 n}^{*}, \\
& F_{1 n}^{*}=-\left.\frac{\partial\left\{G_{n}^{\prime}\left[\Omega_{n}(\theta)\right]^{-1} G_{n}\right\}^{-1}}{\partial \theta^{\prime}}\right|_{\theta=\theta_{1}} G_{n}^{\prime}\left[\Omega_{n}\left(\theta_{1}\right)\right]^{-1} g_{n}\left(\theta_{2}\right), \\
& F_{2 n}^{*}=-\left.\left\{G_{n}^{\prime}\left[\Omega_{n}\left(\theta_{1}\right)\right]^{-1} G_{n}\right\}^{-1} \frac{\partial G_{n}^{\prime}\left[\Omega_{n}(\theta)\right]^{-1} g_{n}\left(\theta_{2}\right)}{\partial \theta^{\prime}}\right|_{\theta=\theta_{1}} .
\end{aligned}
$$

Note that $F_{1 n}^{*}=O_{p}\left(n^{-1 / 2}\right)$ because $G_{n}^{\prime}\left[\Omega_{n}\left(\theta_{1}\right)\right]^{-1} g_{n}\left(\theta_{2}\right)=O_{p}\left(n^{-1 / 2}\right)$ but $F_{2}^{*}=O_{p}\left(\left\|g_{n}\left(\theta_{2}\right)\right\|\right)=$ $O_{p}(1)$ under misspecification. To further expand the FOC of the two-step GMM we observe that $D_{n}^{*}=D^{*}+O_{p}\left(n^{-1 / 2}\right)$ where

$$
\begin{aligned}
D^{*}[., j] & =\left.\left(G^{\prime} \Omega_{1} G\right)^{-1} G^{\prime} \Omega_{1}^{-1} \frac{\partial \Omega(\theta)}{\partial \theta_{[j]}}\right|_{\theta=\theta_{1}} \Omega_{1}^{-1} g_{2}, \\
\frac{\partial \Omega(\theta)}{\partial \theta_{[j]}} & =E\left[g\left(X_{i}, \theta\right) \frac{\partial g\left(X_{i}, \theta\right)^{\prime}}{\partial \theta_{[j]}}\right]+E\left[\frac{\partial g\left(X_{i}, \theta\right)}{\partial \theta_{[j]}} g\left(X_{i}, \theta\right)^{\prime}\right] .
\end{aligned}
$$

Thus,

$$
\begin{aligned}
\sqrt{n}\left(\hat{\theta}_{2}-\theta_{2}\right)= & -\left(G^{\prime} \Omega_{1}^{-1} G\right)^{-1}\left(G^{\prime} \Omega_{1}^{-1} \sqrt{n} g_{n}\left(\theta_{2}\right)+\sqrt{n} G_{n}^{\prime} \Omega_{1}^{-1} g_{2}-G^{\prime} \Omega_{1}^{-1} \sqrt{n} \Omega_{n}\left(\theta_{1}\right) \Omega_{1}^{-1} g_{2}\right) \\
& +D^{*} \sqrt{n}\left(\hat{\theta}_{1}-\theta_{1}\right)+O_{p}\left(n^{-1 / 2}\right) \\
= & -\left(G^{\prime} \Omega_{1}^{-1} G\right)^{-1}\left(G^{\prime} \Omega_{1}^{-1} \sqrt{n} g_{n}\left(\theta_{2}\right)+\sqrt{n} G_{n}^{\prime} \Omega_{1}^{-1} g_{2}-G^{\prime} \Omega_{1}^{-1} \sqrt{n} \Omega_{n}\left(\theta_{1}\right) \Omega_{1}^{-1} g_{2}\right) \\
& -D^{*}\left(G^{\prime} W^{-1} G\right)^{-1}\left(G^{\prime} W^{-1} \sqrt{n} g_{n}\left(\theta_{1}\right)+\sqrt{n} G_{n}^{\prime} W^{-1} g_{1}-G^{\prime} W^{-1} \sqrt{n} W_{n} W^{-1} g_{1}\right) \\
& +O_{p}\left(n^{-1 / 2}\right) .
\end{aligned}
$$

Let

$$
\begin{aligned}
& m_{1 i}=G^{\prime} W^{-1} g\left(X_{i}, \theta_{1}\right)+G\left(X_{i}\right)^{\prime} W^{-1} g_{1}-G^{\prime} W^{-1} W_{i} W^{-1} g_{1}, \\
& m_{2 i}=G^{\prime} \Omega_{1}^{-1} g\left(X_{i}, \theta_{2}\right)+G\left(X_{i}\right)^{\prime} \Omega_{1}^{-1} g_{2}-G^{\prime} \Omega_{1}^{-1} \Omega_{i}\left(\theta_{1}\right) \Omega_{1}^{-1} g_{2}
\end{aligned}
$$

where $W_{n}=n^{-1} \sum_{i=1}^{n} W_{i}$ and $\Omega_{i}(\theta)=g\left(X_{i}, \theta\right) g\left(X_{i}, \theta\right)^{\prime}$. Since $E\left[m_{1 i}\right]=E\left[m_{2 i}\right]=0$, by the multivariate Lindeberg-Lévy CLT,

$$
\sqrt{n}\left(\hat{\theta}_{2}-\theta_{2}\right) \stackrel{d}{\rightarrow} N\left(0, V_{2}\right)
$$


where

$$
\begin{aligned}
V_{2}= & \left(G^{\prime} \Omega_{1}^{-1} G\right)^{-1} E\left[m_{2 i} m_{2 i}^{\prime}\right]\left(G^{\prime} \Omega_{1}^{-1} G\right)^{-1}+D^{*}\left(G^{\prime} W^{-1} G\right)^{-1} E\left[m_{1 i} m_{2 i}^{\prime}\right]\left(G^{\prime} \Omega_{1}^{-1} G\right)^{-1} \\
& +\left(G^{\prime} \Omega_{1}^{-1} G\right)^{-1} E\left[m_{2 i} m_{1 i}^{\prime}\right]\left(G^{\prime} W^{-1} G\right)^{-1} D^{*^{\prime}}+D^{*}\left(G^{\prime} W^{-1} G\right)^{-1} E\left[m_{1 i} m_{1 i}^{\prime}\right]\left(G^{\prime} W^{-1} G\right)^{-1} D^{*^{\prime}} .
\end{aligned}
$$

By replacing the population quantities (expectations) of $V$ with the sample means, we directly obtain $\widehat{V}_{d c}\left(\hat{\theta}_{2}\right)$. By the LLN, $\widehat{V}_{d c}\left(\hat{\theta}_{2}\right) \stackrel{p}{\rightarrow} V_{2}$.

Similarly we have

$$
\sqrt{n}\left(\hat{\theta}_{1}-\theta_{1}\right) \stackrel{d}{\rightarrow} N\left(0, V_{1}\right)
$$

where

$$
V_{1}=\left(G^{\prime} W^{-1} G\right)^{-1} E\left[m_{1 i} m_{1 i}^{\prime}\right]\left(G^{\prime} W^{-1} G\right)^{-1} .
$$

By the LLN, $\widehat{V}_{d c}\left(\hat{\theta}_{1}\right) \stackrel{p}{\rightarrow} V_{1}$. This completes the proof. 


\section{References}

[1] Andrews, D. W. K. (2002). Higher-order improvements of a computationally attractive k-step bootstrap for extremum estimators. Econometrica, 70, 119-162.

[2] Andrews, I. (2019). On the structure of IV estimands. Journal of Econometrics, 211(1), 294-307.

[3] Andrews, I., Gentzkow, M., Shapiro, J.M. (2017). Measuring the sensitivity of parameter estimates to estimation moments. Quarterly Journal of Economics, 132(4), 1553-1592.

[4] Arellano, M., and Bond, S. (1991). Some tests of specification for panel data: Monte Carlo evidence and an application to employment equations. Review of Economic Studies, 58, 277297.

[5] Arellano, M., and Bover, O. (1995). Another look at the instrumental variable estimation of error-components models. Journal of Econometrics, 68, 29-51.

[6] Armstrong, T., and Kolesár, M. (2019). Sensitivity analysis using approximate moment condition models. arXiv preprint arXiv:1808.07387.

[7] Bekker, P. A. (1994). Alternative approximations to the distributions of instrumental variables estimators. Econometrica, 63, 657-681.

[8] Blundell, R., and Bond, S. (1998). Initial conditions and moment restrictions in dynamic panel data models. Journal of Econometrics, 87, 115-143.

[9] Bond, S., and Windmeijer, F. (2005). Reliable inference for GMM estimators? Finite sample properties of alternative test procedures in linear panel data models. Econometric Reviews, $24(1), 1-37$.

[10] Bonhomme, S., and Weidner, M. (2018). Minimizing sensitivity to model misspecification. arXiv preprint arXiv:1807.02161.

[11] Conley, T.G., Hansen, C.B. and Rossi, P.E. (2012). Plausibly exogenous. Review of Economics and Statistics, 94(1), pp.260-272.

[12] Evdokimov, K., and Kolesár, M. (2018). Inference in instrumental variable regression analysis with heterogeneous treatment effects. Working paper.

[13] Guggenberger, P. (2012). On the asymptotic size distortion of tests when instruments locally violate the exogeneity assumption. Econometric Theory, 28(2), 387-421.

[14] Hall, A. R. (2000). Covariance matrix estimation and the power of the overidentifying restrictions test. Econometrica, 68(6), 1517-1527.

[15] Hall, A. R., and Inoue, A. (2003). The large sample behavior of the generalized method of moments estimator in misspecified models. Journal of Econometrics, 114(2), 361-394. 
[16] Hall, P. (1992). The Bootstrap and Edgeworth expansion, New York: Springer-Verlag.

[17] Hall, P. and J. L. Horowitz (1996). Bootstrap critical values for tests based on generalizedmethod-of-moments estimators. Econometrica 64, 891-916.

[18] Han, C. and Phillips, P.C. (2006). GMM with many moment conditions. Econometrica, 74(1), 147-192.

[19] Hansen, B. E. (2006). Edgeworth expansions for the Wald and GMM statistics for nonlinear restrictions. Econometric theory and practice: frontiers of analysis and applied research, ed. by D. Corbae, SN Durlauf, and BE Hansen, Cambridge, 9-35.

[20] Hansen, B. E. (2019). Econometrics. Unpublished Manuscript. University of Wisconsin.

[21] Hansen, B. E., and Lee, S. (2019). Inference for iterated GMM under misspecification. Working paper.

[22] Hansen, L. P. (1982). Large sample properties of generalized method of moments estimators. Econometrica, 50(4), 1029-1054.

[23] Hansen, L. P., Heaton, J., and Yaron, A. (1996). Finite-sample properties of some alternative GMM estimators. Journal of Business 83 Economic Statistics, 14(3), 262-280.

[24] Hwang, J., and Sun, Y. (2018). Should we go one step further? An accurate comparison of one-step and two-step procedures in a generalized method of moments framework. Journal of Econometrics, 207(2), 381-405.

[25] Kitamura, Y., Otsu, T., and Evdokimov, K. (2013). Robustness, infinitesimal neighborhoods, and moment restrictions. Econometrica, 81(3), 1185-1201.

[26] Kundhi, G., and Rilstone, P. (2013). Edgeworth and saddlepoint expansions for nonlinear estimators. Econometric Theory, 29(5), 1057-1078.

[27] Lee, S. (2014). Asymptotic refinements of a misspecification-robust bootstrap for generalized method of moments estimators. Journal of Econometrics, 178, pp.398-413.

[28] Lee, S. (2016). Asymptotic refinements of a misspecification-robust bootstrap for GEL estimators. Journal of Econometrics, 192(1), 86-104.

[29] Lee, S. (2018). A consistent variance estimator for 2sls when instruments identify different LATEs. Journal of Business \&s Economic Statistics, 36(3), 400-410.

[30] Magdalinos, M. A. (1992). Stochastic expansions and asymptotic approximations. Econometric Theory, 8(3), 343-367.

[31] Maasoumi, E., and Phillips, P. C. (1982). On the behavior of inconsistent instrumental variable estimators. Journal of Econometrics, 19(2), 183-201. 
[32] Newey, W. K. (1985). Generalized method of moments specification testing. Journal of Econometrics, 29(3), 229-256.

[33] Newey, W. K., and McFadden, D. (1994). Large sample estimation and hypothesis testing. Handbook of econometrics, 4, 2111-2245.

[34] Newey, W. K., and Smith, R. J. (2004). Higher order properties of GMM and generalized empirical likelihood estimators. Econometrica, 72(1), 219-255.

[35] Newey, W. K., and Windmeijer, F. (2009). Generalized method of moments with many weak moment conditions. Econometrica, 77, 687-719.

[36] Otsu, T. (2011). Moderate deviations of generalized method of moments and empirical likelihood estimators. Journal of Multivariate Analysis, 102(8), 1203-1216.

[37] Rotemberg, J. J. (1983). Instrumental variable estimation of misspecified models. Working paper, MIT Sloan.

[38] Rothenberg, T. J. (1984). Approximating the distributions of econometric estimators and test statistics. Handbook of econometrics, 2, 881-935.

[39] Schennach, S. M. (2007). Point estimation with exponentially tilted empirical likelihood. The Annals of Statistics, 35(2), 634-672.

[40] Windmeijer, F. (2000). A finite sample correction for the variance of linear efficient two-step GMM estimators. IFS Working papers W00/19, Institute for Fiscal Studies.

[41] Windmeijer, F. (2005). A finite sample correction for the variance of linear efficient two-step GMM estimators. Journal of Econometrics, 126(1), 25-51. 


\begin{tabular}{|c|c|c|c|c|c|c|c|}
\hline & $\alpha_{0}$ & 0 & 0.2 & 0.4 & 0.6 & 0.8 & 1 \\
\hline \multirow[t]{14}{*}{$n=50$} & $\hat{\beta}_{1}$ & 1.0833 & 1.0859 & 1.0823 & 1.0833 & 1.0840 & 1.0811 \\
\hline & sd $\hat{\beta}_{1}$ & 0.3229 & 0.3226 & 0.3295 & 0.3417 & 0.3503 & 0.3746 \\
\hline & se $\hat{\beta}_{1}$ & 0.2962 & 0.2963 & 0.2985 & 0.3022 & 0.3047 & 0.3113 \\
\hline & $\mathrm{se}_{d c} \hat{\beta}_{1}$ & 0.3346 & 0.3363 & 0.3417 & 0.3516 & 0.3619 & 0.3794 \\
\hline & $\hat{\beta}_{2}$ & 1.0736 & 1.0656 & 1.0517 & 1.0421 & 1.0344 & 1.0243 \\
\hline & sd $\hat{\beta}_{2}$ & 0.3029 & 0.3035 & 0.3142 & 0.3293 & 0.3470 & 0.3753 \\
\hline & se $\hat{\beta}_{2}$ & 0.2544 & 0.2549 & 0.2575 & 0.2616 & 0.2646 & 0.2720 \\
\hline & $\operatorname{se}_{w} \hat{\beta}_{2}$ & 0.2889 & 0.2900 & 0.2945 & 0.3039 & 0.3133 & 0.3281 \\
\hline & $\operatorname{se}_{d c} \hat{\beta}_{2}$ & 0.3101 & 0.3144 & 0.3231 & 0.3398 & 0.3570 & 0.3813 \\
\hline & $\hat{\beta}_{i t e r}$ & 1.0778 & 1.0672 & 1.0519 & 1.0390 & 1.0290 & 1.0154 \\
\hline & sd $\hat{\beta}_{i t e r}$ & 0.3026 & 0.3028 & 0.3147 & 0.3312 & 0.3517 & 0.3827 \\
\hline & se $\hat{\beta}_{i t e r}$ & 0.2513 & 0.2531 & 0.2573 & 0.2631 & 0.2680 & 0.2766 \\
\hline & $\mathrm{se}_{w} \hat{\beta}_{i t e r}$ & 0.2850 & 0.2859 & 0.2919 & 0.3028 & 0.3140 & 0.3316 \\
\hline & $\operatorname{se}_{d c} \hat{\beta}_{i t e r}$ & 0.3069 & 0.3086 & 0.3176 & 0.3340 & 0.3500 & 0.3742 \\
\hline \multirow[t]{14}{*}{$n=100$} & $\hat{\beta}_{1}$ & 1.0411 & 1.0408 & 1.0413 & 1.0411 & 1.0402 & 1.0412 \\
\hline & sd $\hat{\beta}_{1}$ & 0.2326 & 0.2315 & 0.2337 & 0.2373 & 0.2420 & 0.2477 \\
\hline & se $\hat{\beta}_{1}$ & 0.2212 & 0.2212 & 0.2218 & 0.2229 & 0.2240 & 0.2259 \\
\hline & $\operatorname{se}_{d c} \hat{\beta}_{1}$ & 0.2354 & 0.2359 & 0.2380 & 0.2414 & 0.2458 & 0.2519 \\
\hline & $\hat{\beta}_{2}$ & 1.0353 & 1.0238 & 1.0133 & 1.0041 & 0.9940 & 0.9860 \\
\hline & sd $\hat{\beta}_{2}$ & 0.2153 & 0.2138 & 0.2187 & 0.2239 & 0.2316 & 0.2400 \\
\hline & se $\hat{\beta}_{2}$ & 0.1956 & 0.1957 & 0.1964 & 0.1977 & 0.1991 & 0.2010 \\
\hline & $\operatorname{se}_{w} \hat{\beta}_{2}$ & 0.2089 & 0.2087 & 0.2099 & 0.2130 & 0.2169 & 0.2221 \\
\hline & $\mathrm{se}_{d c} \hat{\beta}_{2}$ & 0.2135 & 0.2143 & 0.2179 & 0.2239 & 0.2315 & 0.2408 \\
\hline & $\hat{\beta}_{\text {iter }}$ & 1.0386 & 1.0260 & 1.0145 & 1.0044 & 0.9931 & 0.9836 \\
\hline & sd $\hat{\beta}_{i t e r}$ & 0.2143 & 0.2126 & 0.2175 & 0.2228 & 0.2311 & 0.2398 \\
\hline & se $\hat{\beta}_{i t e r}$ & 0.1946 & 0.1958 & 0.1978 & 0.2000 & 0.2024 & 0.2053 \\
\hline & $\mathrm{se}_{w} \hat{\beta}_{i t e r}$ & 0.2073 & 0.2079 & 0.2101 & 0.2140 & 0.2187 & 0.2248 \\
\hline & $\mathrm{se}_{d c} \hat{\beta}_{i t e r}$ & 0.2123 & 0.2129 & 0.2164 & 0.2226 & 0.2298 & 0.2392 \\
\hline \multirow[t]{14}{*}{$n=500$} & $\hat{\beta}_{1}$ & 1.0081 & 1.0080 & 1.0085 & 1.0080 & 1.0082 & 1.0085 \\
\hline & sd $\hat{\beta}_{1}$ & 0.1044 & 0.1048 & 0.1047 & 0.1050 & 0.1056 & 0.1061 \\
\hline & se $\hat{\beta}_{1}$ & 0.1035 & 0.1036 & 0.1036 & 0.1038 & 0.1037 & 0.1038 \\
\hline & $\operatorname{se}_{d c} \hat{\beta}_{1}$ & 0.1048 & 0.1049 & 0.1050 & 0.1055 & 0.1057 & 0.1062 \\
\hline & $\hat{\beta}_{2}$ & 1.0066 & 1.0005 & 0.9949 & 0.9885 & 0.9828 & 0.9778 \\
\hline & sd $\hat{\beta}_{2}$ & 0.0962 & 0.0966 & 0.0969 & 0.0970 & 0.0981 & 0.0989 \\
\hline & se $\hat{\beta}_{2}$ & 0.0946 & 0.0946 & 0.0946 & 0.0948 & 0.0948 & 0.0949 \\
\hline & $\mathrm{se}_{w} \hat{\beta}_{2}$ & 0.0958 & 0.0957 & 0.0956 & 0.0958 & 0.0958 & 0.0962 \\
\hline & $\operatorname{se}_{d c} \hat{\beta}_{2}$ & 0.0955 & 0.0956 & 0.0958 & 0.0964 & 0.0970 & 0.0979 \\
\hline & $\hat{\beta}_{i t e r}$ & 1.0074 & 1.0012 & 0.9955 & 0.9891 & 0.9833 & 0.9782 \\
\hline & sd $\hat{\beta}_{i t e r}$ & 0.0960 & 0.0964 & 0.0966 & 0.0968 & 0.0977 & 0.0985 \\
\hline & se $\hat{\beta}_{i t e r}$ & 0.0945 & 0.0949 & 0.0951 & 0.0956 & 0.0959 & 0.0962 \\
\hline & $\mathrm{se}_{w} \hat{\beta}_{i t e r}$ & 0.0957 & 0.0958 & 0.0959 & 0.0964 & 0.0966 & 0.0972 \\
\hline & $\mathrm{se}_{d c} \hat{\beta}_{i t e r}$ & 0.0954 & 0.0954 & 0.0956 & 0.0962 & 0.0967 & 0.0975 \\
\hline
\end{tabular}

Table 1: Monte Carlo Results for Linear IV: $n=50,100,500$ 


\begin{tabular}{|c|c|c|c|c|c|c|c|c|}
\hline & $\alpha_{0}$ & 0 & 0.05 & 0.1 & 0.15 & 0.2 & 0.25 & 0.3 \\
\hline$N=100$ & $\hat{\rho}_{1}$ & 0.4256 & 0.4356 & 0.4571 & 0.4925 & 0.5397 & 0.5926 & 0.6473 \\
\hline \multirow[t]{13}{*}{$T=4$} & $\operatorname{sd} \hat{\rho}_{1}$ & 0.3324 & 0.3376 & 0.3268 & 0.3173 & 0.3107 & 0.3154 & 0.2763 \\
\hline & se $\hat{\rho}_{1}$ & 0.3211 & 0.3215 & 0.3130 & 0.3023 & 0.2899 & 0.2775 & 0.2544 \\
\hline & $\mathrm{se}_{d c} \hat{\rho}_{1}$ & 0.3515 & 0.3520 & 0.3431 & 0.3327 & 0.3196 & 0.3081 & 0.2815 \\
\hline & $\hat{\rho}_{2}$ & 0.4256 & 0.4350 & 0.4554 & 0.4894 & 0.5351 & 0.5876 & 0.6420 \\
\hline & sd $\hat{\rho}_{2}$ & 0.3502 & 0.3552 & 0.3443 & 0.3362 & 0.3327 & 0.3259 & 0.2966 \\
\hline & se $\hat{\rho}_{2}$ & 0.3113 & 0.3115 & 0.3032 & 0.2929 & 0.2806 & 0.2680 & 0.2466 \\
\hline & $\mathrm{se}_{w} \hat{\rho}_{2}$ & 0.3376 & 0.3377 & 0.3300 & 0.3208 & 0.3087 & 0.2961 & 0.2734 \\
\hline & $\mathrm{se}_{d c} \hat{\rho}_{2}$ & 0.3684 & 0.3673 & 0.3594 & 0.3521 & 0.3375 & 0.3258 & 0.3027 \\
\hline & $\hat{\rho}$ & 0.4182 & 0.4276 & 0.4467 & 0.4790 & 0.5234 & 0.5736 & 0.6267 \\
\hline & $\mathrm{sd} \hat{\rho}$ & 0.3656 & 0.3702 & 0.3619 & 0.3574 & 0.3568 & 0.3551 & 0.3324 \\
\hline & se $\hat{\rho}$ & 0.3123 & 0.3122 & 0.3038 & 0.2938 & 0.2819 & 0.2688 & 0.2482 \\
\hline & $\operatorname{se}_{w} \hat{\rho}$ & 0.3483 & 0.3489 & 0.3427 & 0.3350 & 0.3243 & 0.3133 & 0.2909 \\
\hline & $\operatorname{se}_{d c} \hat{\rho}$ & 0.3756 & 0.3773 & 0.3695 & 0.3619 & 0.3495 & 0.3398 & 0.3122 \\
\hline$N=100$ & $\hat{\rho}_{1}$ & 0.4234 & 0.4272 & 0.4398 & 0.4626 & 0.4992 & 0.5460 & 0.6008 \\
\hline \multirow[t]{13}{*}{$T=6$} & $\mathrm{sd} \hat{\rho}_{1}$ & 0.1469 & 0.1471 & 0.1468 & 0.1480 & 0.1493 & 0.1503 & 0.1477 \\
\hline & se $\hat{\rho}_{1}$ & 0.1458 & 0.1455 & 0.1441 & 0.1418 & 0.1377 & 0.1308 & 0.1221 \\
\hline & $\mathrm{se}_{d c} \hat{\rho}_{1}$ & 0.1537 & 0.1540 & 0.1542 & 0.1546 & 0.1545 & 0.1509 & 0.1441 \\
\hline & $\hat{\rho}_{2}$ & 0.4217 & 0.4249 & 0.4363 & 0.4570 & 0.4909 & 0.5351 & 0.5891 \\
\hline & $\mathrm{sd} \hat{\rho}_{2}$ & 0.1630 & 0.1640 & 0.1650 & 0.1667 & 0.1704 & 0.1727 & 0.1708 \\
\hline & se $\hat{\rho}_{2}$ & 0.1327 & 0.1324 & 0.1310 & 0.1284 & 0.1242 & 0.1175 & 0.1094 \\
\hline & $\mathrm{se}_{w} \hat{\rho}_{2}$ & 0.1635 & 0.1634 & 0.1631 & 0.1626 & 0.1611 & 0.1567 & 0.1493 \\
\hline & $\mathrm{se}_{d c} \hat{\rho}_{2}$ & 0.1628 & 0.1634 & 0.1646 & 0.1668 & 0.1693 & 0.1687 & 0.1643 \\
\hline & $\hat{\rho}$ & 0.4167 & 0.4194 & 0.4294 & 0.4476 & 0.4762 & 0.5137 & 0.5606 \\
\hline & $\operatorname{sd} \hat{\rho}$ & 0.1782 & 0.1799 & 0.1831 & 0.1872 & 0.1972 & 0.2065 & 0.2127 \\
\hline & se $\hat{\rho}$ & 0.1328 & 0.1325 & 0.1312 & 0.1289 & 0.1250 & 0.1191 & 0.1119 \\
\hline & $\mathrm{se}_{w} \hat{\rho}$ & 0.1778 & 0.1783 & 0.1794 & 0.1822 & 0.1858 & 0.1887 & 0.1887 \\
\hline & $\operatorname{se}_{d c} \hat{\rho}$ & 0.1773 & 0.1784 & 0.1806 & 0.1855 & 0.1916 & 0.1965 & 0.1975 \\
\hline
\end{tabular}

Table 2: Monte Carlo Results for Linear Dynamic Panel: $N=100$ and $T=4,6$ 


\begin{tabular}{|c|c|c|c|c|c|c|c|c|}
\hline & $\alpha_{0}$ & 0 & 0.05 & 0.1 & 0.15 & 0.2 & 0.25 & 0.3 \\
\hline$N=500$ & $\hat{\rho}_{1}$ & 0.4879 & 0.4938 & 0.5170 & 0.5531 & 0.5990 & 0.6502 & 0.7016 \\
\hline \multirow[t]{13}{*}{$T=4$} & $\operatorname{sd} \hat{\rho}_{1}$ & 0.1379 & 0.1363 & 0.1330 & 0.1284 & 0.1220 & 0.1131 & 0.1044 \\
\hline & se $\hat{\rho}_{1}$ & 0.1369 & 0.1356 & 0.1322 & 0.1265 & 0.1192 & 0.1107 & 0.1020 \\
\hline & $\mathrm{se}_{d c} \hat{\rho}_{1}$ & 0.1389 & 0.1377 & 0.1347 & 0.1292 & 0.1222 & 0.1136 & 0.1047 \\
\hline & $\hat{\rho}_{2}$ & 0.4893 & 0.4950 & 0.5180 & 0.5539 & 0.6001 & 0.6517 & 0.7034 \\
\hline & sd $\hat{\rho}_{2}$ & 0.1400 & 0.1385 & 0.1357 & 0.1314 & 0.1254 & 0.1164 & 0.1075 \\
\hline & se $\hat{\rho}_{2}$ & 0.1361 & 0.1348 & 0.1315 & 0.1258 & 0.1186 & 0.1101 & 0.1014 \\
\hline & $\mathrm{se}_{w} \hat{\rho}_{2}$ & 0.1389 & 0.1377 & 0.1349 & 0.1295 & 0.1225 & 0.1138 & 0.1046 \\
\hline & $\mathrm{se}_{d c} \hat{\rho}_{2}$ & 0.1404 & 0.1393 & 0.1368 & 0.1318 & 0.1251 & 0.1165 & 0.1072 \\
\hline & $\hat{\rho}$ & 0.4891 & 0.4948 & 0.5178 & 0.5537 & 0.5999 & 0.6515 & 0.7032 \\
\hline & $\mathrm{sd} \hat{\rho}$ & 0.1403 & 0.1389 & 0.1362 & 0.1319 & 0.1260 & 0.1170 & 0.1080 \\
\hline & se $\hat{\rho}$ & 0.1362 & 0.1349 & 0.1315 & 0.1259 & 0.1187 & 0.1102 & 0.1016 \\
\hline & $\operatorname{se}_{w} \hat{\rho}$ & 0.1393 & 0.1382 & 0.1354 & 0.1301 & 0.1232 & 0.1145 & 0.1052 \\
\hline & $\operatorname{se}_{d c} \hat{\rho}$ & 0.1408 & 0.1397 & 0.1373 & 0.1323 & 0.1256 & 0.1170 & 0.1077 \\
\hline$N=500$ & $\hat{\rho}_{1}$ & 0.4835 & 0.4869 & 0.4997 & 0.5237 & 0.5621 & 0.6130 & 0.6687 \\
\hline \multirow[t]{13}{*}{$T=6$} & $\mathrm{sd} \hat{\rho}_{1}$ & 0.0691 & 0.0691 & 0.0689 & 0.0681 & 0.0676 & 0.0654 & 0.0615 \\
\hline & se $\hat{\rho}_{1}$ & 0.0690 & 0.0688 & 0.0680 & 0.0664 & 0.0637 & 0.0595 & 0.0544 \\
\hline & $\mathrm{se}_{d c} \hat{\rho}_{1}$ & 0.0698 & 0.0697 & 0.0695 & 0.0691 & 0.0681 & 0.0656 & 0.0611 \\
\hline & $\hat{\rho}_{2}$ & 0.4842 & 0.4875 & 0.4998 & 0.5227 & 0.5595 & 0.6089 & 0.6639 \\
\hline & $\mathrm{sd} \hat{\rho}_{2}$ & 0.0712 & 0.0714 & 0.0718 & 0.0723 & 0.0735 & 0.0726 & 0.0689 \\
\hline & se $\hat{\rho}_{2}$ & 0.0677 & 0.0675 & 0.0667 & 0.0651 & 0.0623 & 0.0581 & 0.0530 \\
\hline & $\mathrm{se}_{w} \hat{\rho}_{2}$ & 0.0711 & 0.0711 & 0.0710 & 0.0708 & 0.0701 & 0.0678 & 0.0634 \\
\hline & $\mathrm{se}_{d c} \hat{\rho}_{2}$ & 0.0708 & 0.0709 & 0.0713 & 0.0722 & 0.0730 & 0.0720 & 0.0682 \\
\hline & $\hat{\rho}$ & 0.4841 & 0.4874 & 0.4996 & 0.5223 & 0.5585 & 0.6066 & 0.6603 \\
\hline & $\operatorname{sd} \hat{\rho}$ & 0.0715 & 0.0718 & 0.0723 & 0.0732 & 0.0752 & 0.0757 & 0.0737 \\
\hline & se $\hat{\rho}$ & 0.0677 & 0.0675 & 0.0667 & 0.0651 & 0.0624 & 0.0583 & 0.0534 \\
\hline & $\mathrm{se}_{w} \hat{\rho}$ & 0.0715 & 0.0715 & 0.0715 & 0.0717 & 0.0720 & 0.0713 & 0.0685 \\
\hline & $\operatorname{se}_{d c} \hat{\rho}$ & 0.0712 & 0.0713 & 0.0718 & 0.0730 & 0.0746 & 0.0749 & 0.0725 \\
\hline
\end{tabular}

Table 3: Monte Carlo Results for Linear Dynamic Panel: $N=500$ and $T=4,6$ 


\begin{tabular}{|c|c|c|c|c|c|}
\hline & $\gamma_{0}$ & 0 & 0.025 & 0.05 & 0.1 \\
\hline$N=100$ & $\hat{\beta}_{1}$ & 0.9793 & 0.9534 & 0.9268 & 0.8744 \\
\hline \multirow[t]{13}{*}{$T=4$} & $\operatorname{sd} \hat{\beta}_{1}$ & 0.1521 & 0.1529 & 0.1538 & 0.1588 \\
\hline & se $\hat{\beta}_{1}$ & 0.1469 & 0.1468 & 0.1465 & 0.1472 \\
\hline & $\mathrm{se}_{d c} \hat{\beta}_{1}$ & 0.1546 & 0.1555 & 0.1563 & 0.1604 \\
\hline & $\hat{\beta}_{2}$ & 0.9849 & 0.9588 & 0.9322 & 0.8773 \\
\hline & sd $\hat{\beta}_{2}$ & 0.1404 & 0.1422 & 0.1451 & 0.1552 \\
\hline & se $\hat{\beta}_{2}$ & 0.1243 & 0.1244 & 0.1242 & 0.1253 \\
\hline & $\operatorname{se}_{w} \hat{\beta}_{2}$ & 0.1390 & 0.1402 & 0.1415 & 0.1473 \\
\hline & $\mathrm{se}_{d c} \hat{\beta}_{2}$ & 0.1343 & 0.1366 & 0.1390 & 0.1482 \\
\hline & $\hat{\beta}$ & 0.9858 & 0.9599 & 0.9334 & 0.8781 \\
\hline & $\operatorname{sd} \hat{\beta}$ & 0.1417 & 0.1439 & 0.1474 & 0.1600 \\
\hline & se $\hat{\beta}$ & 0.1243 & 0.1244 & 0.1242 & 0.1253 \\
\hline & $\operatorname{se}_{w} \hat{\beta}$ & 0.1393 & 0.1408 & 0.1426 & 0.1507 \\
\hline & $\mathrm{se}_{d c} \hat{\beta}$ & 0.1352 & 0.1378 & 0.1406 & 0.1517 \\
\hline \multirow{14}{*}{$\begin{array}{c}N=100 \\
T=6\end{array}$} & $\hat{\beta}_{1}$ & 0.9755 & 0.9577 & 0.9411 & 0.9060 \\
\hline & $\operatorname{sd} \hat{\beta}_{1}$ & 0.1027 & 0.1043 & 0.1051 & 0.1083 \\
\hline & se $\hat{\beta}_{1}$ & 0.1002 & 0.1003 & 0.1004 & 0.1013 \\
\hline & $\mathrm{se}_{d c} \hat{\beta}_{1}$ & 0.1056 & 0.1064 & 0.1075 & 0.1107 \\
\hline & $\hat{\beta}_{2}$ & 0.9833 & 0.9649 & 0.9466 & 0.9080 \\
\hline & $\operatorname{sd} \hat{\beta}_{2}$ & 0.0906 & 0.0929 & 0.0948 & 0.1017 \\
\hline & se $\hat{\beta}_{2}$ & 0.0716 & 0.0718 & 0.0720 & 0.0731 \\
\hline & $\operatorname{se}_{w} \hat{\beta}_{2}$ & 0.0905 & 0.0914 & 0.0930 & 0.0978 \\
\hline & $\mathrm{se}_{d c} \hat{\beta}_{2}$ & 0.0836 & 0.0853 & 0.0876 & 0.0944 \\
\hline & $\hat{\beta}$ & 0.9857 & 0.9671 & 0.9484 & 0.9083 \\
\hline & $\operatorname{sd} \hat{\beta}$ & 0.0946 & 0.0970 & 0.0998 & 0.1099 \\
\hline & se $\hat{\beta}$ & 0.0716 & 0.0718 & 0.0720 & 0.0731 \\
\hline & $\operatorname{se}_{w} \hat{\beta}$ & 0.0937 & 0.0952 & 0.0976 & 0.1054 \\
\hline & $\operatorname{se}_{d c} \hat{\beta}$ & 0.0866 & 0.0885 & 0.0916 & 0.1006 \\
\hline
\end{tabular}

Table 4: Monte Carlo Results for Linear Panel Model: $N=100$ and $T=4,6$ 


\begin{tabular}{|c|c|c|c|c|c|}
\hline & $\gamma_{0}$ & 0 & 0.025 & 0.05 & 0.1 \\
\hline$N=500$ & $\hat{\beta}_{1}$ & 0.9958 & 0.9683 & 0.9406 & 0.8853 \\
\hline \multirow[t]{13}{*}{$T=4$} & sd $\hat{\beta}_{1}$ & 0.0685 & 0.0686 & 0.0695 & 0.0716 \\
\hline & se $\hat{\beta}_{1}$ & 0.0679 & 0.0678 & 0.0677 & 0.0679 \\
\hline & $\mathrm{se}_{d c} \hat{\beta}_{1}$ & 0.0686 & 0.0690 & 0.0696 & 0.0716 \\
\hline & $\hat{\beta}_{2}$ & 0.9970 & 0.9710 & 0.9443 & 0.8892 \\
\hline & sd $\hat{\beta}_{2}$ & 0.0652 & 0.0657 & 0.0676 & 0.0730 \\
\hline & se $\hat{\beta}_{2}$ & 0.0632 & 0.0631 & 0.0630 & 0.0634 \\
\hline & $\operatorname{se}_{w} \hat{\beta}_{2}$ & 0.0648 & 0.0653 & 0.0662 & 0.0694 \\
\hline & $\operatorname{se}_{d c} \hat{\beta}_{2}$ & 0.0634 & 0.0643 & 0.0658 & 0.0708 \\
\hline & $\hat{\beta}$ & 0.9970 & 0.9712 & 0.9446 & 0.8897 \\
\hline & $\operatorname{sd} \hat{\beta}$ & 0.0652 & 0.0658 & 0.0678 & 0.0738 \\
\hline & se $\hat{\beta}$ & 0.0632 & 0.0631 & 0.0630 & 0.0634 \\
\hline & $\operatorname{se}_{w} \hat{\beta}$ & 0.0648 & 0.0652 & 0.0662 & 0.0698 \\
\hline & $\mathrm{se}_{d c} \hat{\beta}$ & 0.0634 & 0.0643 & 0.0659 & 0.0715 \\
\hline$N=500$ & $\hat{\beta}_{1}$ & 0.9947 & 0.9758 & 0.9564 & 0.9181 \\
\hline \multirow[t]{13}{*}{$T=6$} & sd $\hat{\beta}_{1}$ & 0.0473 & 0.0476 & 0.0482 & 0.0498 \\
\hline & se $\hat{\beta}_{1}$ & 0.0469 & 0.0470 & 0.0470 & 0.0473 \\
\hline & $\mathrm{se}_{d c} \hat{\beta}_{1}$ & 0.0475 & 0.0479 & 0.0484 & 0.0499 \\
\hline & $\hat{\beta}_{2}$ & 0.9968 & 0.9778 & 0.9582 & 0.9175 \\
\hline & $\operatorname{sd} \hat{\beta}_{2}$ & 0.0432 & 0.0440 & 0.0454 & 0.0494 \\
\hline & se $\hat{\beta}_{2}$ & 0.0408 & 0.0408 & 0.0410 & 0.0414 \\
\hline & $\operatorname{se}_{w} \hat{\beta}_{2}$ & 0.0431 & 0.0436 & 0.0445 & 0.0471 \\
\hline & $\mathrm{se}_{d c} \hat{\beta}_{2}$ & 0.0413 & 0.0421 & 0.0434 & 0.0470 \\
\hline & $\hat{\beta}$ & 0.9969 & 0.9779 & 0.9584 & 0.9174 \\
\hline & $\operatorname{sd} \hat{\beta}$ & 0.0433 & 0.0441 & 0.0457 & 0.0504 \\
\hline & se $\hat{\beta}$ & 0.0408 & 0.0408 & 0.0410 & 0.0414 \\
\hline & $\mathrm{se}_{w} \hat{\beta}$ & 0.0431 & 0.0437 & 0.0447 & 0.0479 \\
\hline & $\mathrm{se}_{d c} \hat{\beta}$ & 0.0414 & 0.0423 & 0.0437 & 0.0479 \\
\hline
\end{tabular}

Table 5: Monte Carlo Results for Linear Panel Model: $N=500$ and $T=4,6$ 